\title{
Qualitative Analysis and Dynamical Behavior of a Lassa Haemorrhagic Fever Model with Exposed Rodents and Saturated Incidence Rate
}

\section{ADEDAPO CHRIS LOYINMI ( $\square$ loyinmiac@tasued.edu.ng )}

Tai Solarin University of Education, ljagun, ljebu-ode, Ogun state, Nigeria https://orcid.org/0000-00026171-4256

\section{KINGSLEY TIMILEHIN AKINFE ( $\nabla$ akinfetimilehin@gmail.com )}

Tai Solarin University of Education, ljagun, ljebu-Ode, Ogun state, Nigeria https://orcid.org/0000-00025308-7053

\section{ABISOYE ABIODUN OJO}

Tai Solarin University of Education, ljagun, ljebu-Ode, Ogun state, Nigeria

\section{Research Article}

Keywords: Lassa Haemorrhagic fever, saturated incidence rate, stability analysis, sensitivity analysis, Lassa Haemorrhagic fever (LHF) free equilibrium, numerical simulation, effective contact rates, nonlinear force of infection

Posted Date: June 8th, 2020

DOI: https://doi.org/10.21203/rs.3.rs-33293/v1

License: (c) (1) This work is licensed under a Creative Commons Attribution 4.0 International License. Read Full License

Version of Record: A version of this preprint was published at Scientific African on October 1st, 2021. See the published version at https://doi.org/10.1016/j.sciaf.2021.e01028. 


\title{
QUALITATIVE ANALYSIS AND DYNAMICAL BEHAVIOR OF A LASSA HAEMORRHAGIC FEVER MODEL WITH EXPOSED RODENTS AND SATURATED INCIDENCE RATE
}

\author{
${ }^{1 .}$ LOYINMI, Adedapo Chris* $\quad{ }^{2}$ AKINFE, Timilehin Kingsley $\quad{ }^{3 .}$ OJO, Abiodun Abisoye \\ ${ }^{1,2,3}$ Department of Mathematics, Tai Solarin University of Education, Ijagun, \\ Ijebu ode, Ogun state, Nigeria \\ 1E-mail: loyinmiac@tasued.edu.ng* $\quad{ }^{2}$ Email: akinfetimilehin@gmail.com \\ ${ }^{1}$ ORCID: 0000-0002-6171-4256 I $\quad{ }^{2}$ ORCID: 0000-0002-5308-7053 \\ ${ }^{3}$ Email: Abiodunabisoye16@yahoo.com \\ "Correspondence: loyinmiac@tasued.edu.ng; akinfetimilehin@gmail.com
}

\begin{abstract}
We have proposed an unprecedented deterministic model of Lassa Haemorrhagic fever (LHF) model with nonlinear force of LHF infection to capture the transmission dynamics and long term effects of the disease. The Qualitative analyses we have conveyed on this model using well established methods viz: Cauchy's differential theorem, Birkhoff \& Rota's theorem verifies and reveals the well-posedness, and carrying capacity of the model respectively. We established that a LHF-free equilibrium termed the disease-free equilibrium (DFE) exists for this model and this equilibrium however from our stability analyses, tends to be stable when the basic reproduction number computed via next generation matrix method is less than unity (one); and unstable if otherwise. Furthermore, we have carried out a sensitivity analyses to check variation effects of the model parameters when increased or decreased using the normalized forward-sensitivity index; unraveling the most sensitive parameters which requires the attention of the healthcare workers as; the effective contact rates $\beta_{1}, \beta_{2}$, and $\beta_{3}$, and the rodents' recruitment rate $\Omega_{R}$. After which numerical simulations of the model were carried out to verify our qualitative analyses
\end{abstract}


(Stability and sensitivity analysis) and to study the dynamical behavior of the model; showing that the presence of saturation instantaneously causes the system to approach a DFE/LHF-Free equilibrium.

From these qualitative analyses and numerical simulation results, we recommend early intervention and early treatment of Lassa haemorrhagic virus infection (LAHV) with Ribavirin on the infected, maximum hygiene practices and periodic evacuation of rodents in households in order to curb the recruitment of wild/rodents.

Keywords: Lassa Haemorrhagic fever, saturated incidence rate, stability analysis, sensitivity analysis, Lassa Haemorrhagic fever (LHF) free equilibrium, numerical simulation, effective contact rates, nonlinear force of infection

\subsection{INTRODUCTION}

Lassa fever (LF) is a zoonotic disease which means that it is being transmitted from animals to humans (animal borne disease). Lassa fever is an acute and deadly infectious illness also known as 'Lassa hemorraghic fever (LHF) caused by a Lassa Virus (LASV) or the Lassa Hemorrhagic fever virus (LASHFV). Lassa fever virus is mainly a zoonosis (an animal-borne disease) being reserved, carried and transmitted by "natal multimammate rat" (Mastomys natalensis) or the African rat [1, 2]. The virus is a single-stranded RNA virus belonging to the virus family Arenaviridae whose members are generally associated with rodent-transmitted diseases in humans. Arenavirus infections are relatively common in humans in some areas of the world and can cause severe illnesses. The virus particles are spherical and have an average diameter of 110130 nanometers. All are enveloped in a lipid (fat) membrane and they show grainy particles that are ribosomes acquired from their host cells when viewed cross-sectionally (CDC, 2014) [3].

Lassa fever was first found in a town called "Lassa" in the Yedseram River Valley in the present Borno state of the Northern Nigeria in 1969 [4]. The first victim is Laura Wine, 65 years old female nurse who works at Lassa Mission Hospital and since its initial discrovery in Lassa, Nigeria, rural and nosocomical outbreaks of Lassa fever have occurred repeatedly in other parts of Nigeria: Jos, Onitsha, Zorikwa, Ekpoma and so on. 
Lassa fever is endemic in Nigeria, Liberia, Sierra Leone, Guinea, and other West African countries, affecting 2-3 million people with 5000-10000 fatalities annually [5]

Pro-Med international society for infectious diseases [6-8] reported outbreaks in some cities of West African countries of Sierra Leone, Liberia, and Guinea. In Cote d'Ivoire, Ghana, Togo and Benin, no outbreaks have ever been recorded, though isolation cases show evidence of viral circulation. However, some Lassa fever cases have been imported in the U.S and U.K through travelers who acquire the disease elsewhere.

Lassa fever is endemic in Nigeria and the annual peak of human cases is usually observed during the dry season (December-April) following the reproduction cycle of the Mastomys rats in the wet season (May-June). However, lassa fever being endemic in Nigeria has made the country develop the capacity to manage its outbreaks of which the current overall risk level is considered moderate at national level (WHO Disease Outbreak News, (2020)) [9].

The natal multimammate rat or African rat is probably the most common mouse in equatorial Africa, common in households and eaten as a delicacy in some areas. The multimammate rat can quickly reproduce a large number of offspring, tends to colonize human settlements increasing the risk of rodent-human contact, and is found throughout the west, central and eastern part of the African continent specifically in Sierra Lione, Guinea, Liberia, Ghana and Nigeria.

Once the rat has become a carrier, it will excrete the virus throughout the rest of its lifetime through its feces and urine creating ample opportunity for exposure [Goeijenbier, Marco et al. (2012)]. The transmission of the virus to humans occurs most commonly through ingestion or inhalation because the Mastomys rodents shed the virus in urine, droppings and direct contact with these materials, through touching soiled objects, eating food grains in the stores, or exposure to open cuts or sores.

Because Mastomys rodents often live in around homes and scavenge on the leftovers, food items, or poorly stored food by the humans; direct contact transmission is common and might surely occur. The Mastomys rodents are sometimes consumed as food source and infection may occur when rodents are caught and prepared. Contact with the Lassa Hemorrhagic fever virus may also occur when an individual inhales tiny particles in the air contaminated with the infected rodent excretions. The aerosol or airborne transmission may occur during cleaning activities, such as sweeping and so on. Direct contact with the infected rodents is not the only way in which people 
are infected; human to human transmission may occur after exposure to virus in the blood, tissue, secretions, or excretions of a Lassa virus-infected individual (CDC) [10].

Casual contact (Including skin-to-skin contact without exchange of body fluids) does not spread lassa virus. Person to person transmission is common in the health care setting (called nosocomical transmission) where proper personal protective equipment (PPE) is not available or not used. Lassa virus may be spread also in contaminated medical equipment, such as reused needles and so on. Unlike Ebola virus, Marburg virus and others, Lassa fever is not as contagious person to person, nor as deadly when compared to these viruses.

The most interesting feature of this virus is how differently it affects pregnant women in comparison with non-pregnant women. When Lassa fever infects pregnant women late in their third trimester, inducing delivery is necessary for mother to have a good chance of survival. This is because the virus has an affinity for the placenta and other highly vascular tissues. The fetus has only a one in ten chance of survival no matter what course of action is taken; hence, the focus is always on saving the life of the mother. Stillbirths or fetal loss occurs in $95 \%$ of pregnancies. Following delivery, women should receive same treatment as other people with lassa fever.

\subsection{SYMPTOMS OF LASSA HEMORRHAGIC FEVER (LHF)}

Signs and symptoms of Lassa fever typically occur 1-3 weeks after the patient comes into contact with the virus. About $80 \%$ of persons infected with Lassa virus are asymptomatic but the remaining $20 \%$, the illness manifests as a febrile illness of variable severity associated with multiple organ dysfunctions with or without haemorrhage [WHO, (2020)].

When symptoms occur they typically include fever, weakness, headaches, vomiting, general malaise, and muscle pains. Less commonly, there may be bleeding from the mouth or gastrointestinal tract. However, the disease may progress to more serious symptoms including hemorrhaging (in gums, eyes, or nose, as examples), respiratory distress, repeated vomiting, and facial swelling, pain in the chest, back, abdomen and shock. Neurological problems have also been described, including hearing loss, tremors, and encephalitis.

The most common complications of Lassa fever is deafness. Various degrees of deafness occur in approximately one-third of infections, and in many cases hearing loss is permanent. As far as 
is known, severity of the disease does not affect this complication: deafness may develop in mild as well as in severe cases.

Approximately $15 \%-20 \%$ of patients hospitalized for Lassa fever die from the illness. However, only $1 \%$ of all Lassa virus infections result in death. The death rates of women in the third trimester of pregnancy are particularly high. Spontaneous abortion is a serious complication of infection with an estimated 95\% mortality in fetuses of infected pregnant mothers [Centre for Disease Control (CDC), (2020)].

The risk of death once infected frequently occurs within two weeks of the onset of symptoms [WHO, (2016)]. Among those who survive about a quarter have hearing loss, which improves within three months in about half of these cases.

Because of the symptoms of Lassa fever are so varied and non-specific, clinical diagnosis is often difficult. Confirmation is by laboratory testing to detect the virus' RNA, antibodies for the virus, or the virus itself in cell culture [WHO, (2016)]. In half of the infected cases, hearing returns partially after 1-3 months of recovery, and transient hairloss and gait disturbance may occur during recovery (World Health Organization, 2017) [11]

Lassa fever is also associated with occasional epidemics, during which the case-fatality rate can reach $50 \%$ in hospitalized patients.

\subsection{THE EPIDEMIOLOGY OF LASSA FEVER IN NIGERIA}

The issue of Lassa Haemorrhagic fever has been very prevalent in Nigeria till date, threatening the well being of humans and their socio-economic life. Outbreaks of Lassa fever virus occurs regularly in West Africa with the most recent one in Nigeria (Amy, 2018) [12]. From $1^{\text {st }}$ January 2018 through $18^{\text {th }}$ March 2018, about 1495 suspected cases and 119 deaths have been reported in Nigeria from 19 states of the federation including Anambra, Bauchi, Benue, Delta, Ebonyi, Edo, Ekiti, FCT, Abuja, Gombe, Imo, Kaduna, Kogi, Lagos, Nasarawa, Ondo, Osun, Plateau, Rivers, and Taraba (WHO, 2018) [13].

During this period, 376 cases were confirmed, 9 were classified probable, 1084 were reported negative and 26 are pending; awaiting laboratory results [13]. Among the 376 confirmed and the 9 classified probable cases, 95 deaths were reported giving a case-fatality rate of the confirmed and probable cases to be $24.7 \%$ [13]. Similar reports about the current ongoing Lassa outbreaks 
and fatality cases in Nigeria can be found in [14-15]. Since 1 January 2018, the number of Lassa fever cases increased from 10 to 70 weekly reported cases [13].

However, since mid-February 2018, there has been a downward trend in the weekly reported

number of cases [13]. The Nigeria Center for Disease Control NCDC has built a laboratory in Ebonyi State that has the equipment needed to identify the Lassa fever virus, which is the fourth of its kind to be established by the institution in the country (Amy, 2018) [12].

Most Recently, In April 5 ${ }^{\text {th }}, 2020$ it was reported that there were 93 suspected cases, 12 confirmed cases and 3 deaths across five (5) states and nine (9) local government areas with $25 \%$ CFR. It was deduced that the predominant age-group affected is 21-30 years (Range: <1 to 78 years, Median age: 33 years) and the male to female ratio for confirmed cases is $1: 1$ [26].

\subsection{TREATMENT OF LASSA HAEMORRAGHIC FEVER}

Ribavirin, an antiviral drug, has been used with success in Lassa fever patients. It has been shown to be most effective when given early in the course of the illness. Patients should also receive supportive care consisting of maintenance of appropriate fluid and electrolyte balance, oxygenation and blood pressure, as well as treatment of any other complicating infections [3, 10].

In curbing the spread or implementing an intervention strategy as regards infectious diseases like the HIV, Ebola, Tuberculosis, Lassa Hemorrhagic fever (LASV) and so on; some pertinent factors needs to be taken into account and consideration viz: the pathogen of the disease, mode and nature of transmission, the agents of transmission, the force of infection, the incident rates, the age-groups in the transmission if any, and so on. Knowing these makes one understand the transmission dynamics of such infectious disease making it hitch free to propose, set-in, implement, or try out an intervention strategy.

A mathematical model is description of the behavior of the system using mathematical concepts and language. The process of developing a mathematical model is known as mathematical modeling. Mathematical models provide a clear picture of the disease's behavior and its current and future impacts on an endemic society. These models are tools of policy and decision makers when taking appropriate control measures on a particular disease. 
A differential equation is a mathematical equation that relates some function with its derivatives. In applications, the functions usually represent physical quantities, the derivatives represent their rates of change, and the equation defines a relationship between the two. The modeling real life phenomenon or system (For example, the transmission of infectious diseases) is incomplete without incorporating differential equations. These two works together simultaneously because mathematical ideas, models, and conjectures must be translated into mathematical equation so as to express their relationships [27-29].

\subsection{INCIDENCE RATES AND FORCES OF INFECTION}

Several authors have employed the following incidence functions: The first one is the bilinear incidence rate $\beta S I$, where $S$ and $I$ are respectively the number of susceptible and infected individuals in the population, and $\beta$ is a positive constant.

The second one is the saturated incidence of the form $\frac{\beta S I}{1+P I}$, where $\mathrm{p}$ is a positive constant, and the saturated incidence of the form $\frac{\beta S I}{1+P S}$.

Less attention has been paid to implementing the saturated incidence rates in epidemic models due to the fact that most researchers make use of the bilinear incidence rate $\beta S I$ and the standard incidence rates $\frac{\beta S I}{N}$. This became the motivation for this research work.

Esteva and Matias (2001) [16], introduced the saturated incidence rate, which tends to a saturation level when $I$ the infected gets very large, $\beta I$ and $\beta S$ at the numerator for the first and second respectively measures the infection force when the disease is entering a fully susceptible

population, $\frac{1}{1+p I}$ and $\frac{1}{1+p S}$ for the first and second function respectively measures the inhibition effect from the behavioral change of the susceptible individuals when their number increases or from the crowding effect of the infective individuals. The effect of the saturation factor or parameter " $p$ " stems from epidemic control (taking appropriate preventive measures). In this case, it could just be any factor hindering the spread of the epidemic, the antibodies developed from the human immune system to combat the disease and so on. This saturated incidence rate is more reasonable than the bilinear incidence rate because it includes the behavioral change and crowding effect of the infective individuals and prevents the unboundedness of the contact rate by choosing suitable parameters. 


\subsection{THEORY OF SENSITIVITY ANALYSIS}

Due to the prevalent nature of the lassa virus, it became a point of duty and necessary to design, evaluate, and implement a control plan in eradicating the ubiquitous nature of the virus. In this case we have to test and check for the sensitive parameters spurring the outbreak in the dynamics of the disease; doing this implies carrying out a sensitivity analysis on the transmission dynamics of the disease.

Sensitivity analysis studies the variation of the outputs of a model caused by variations in the inputs. In essence, sensitivity analysis determines which parameters and initial conditions (Inputs) affect the quantities of interest (outputs) of the model the most. The first reason why this analysis is important is that it tells the researcher which parameters deserve the most numerical attention (Micheal A. Mikucki, (2012)) [17].

A highly sensitive parameter should be carefully estimated, as a small variation in that parameter will lead to large qualitative changes to the quantity of interest and may even produce qualitatively different results.

An insensitive parameter, on the other hand, does not require as much effort to estimate as a small variation in that parameter will not produce large changes to a quality of interest.

The sensitivity analysis is a crucial analysis showing the importance of each parameter in the disease transmission. The value obtained by carrying out sensitive checks on a parameter is referred to as "sensitivity index".

In this research, the sensitivity index of parameters is computed with respect to the reproduction number using the normalized forward sensitivity index.

Several authors have carried out sensitivity analysis on distinct models viz: Samson Olaniyi et al. (2016), J.O Akanni (2017), Akinpelu F.O (2018), Akinade Mary O. (2020) [18-21], to mention a few.

In the quest of understanding the varying dynamical behavior of epidemic models, some authors have also deemed it fit to study the various type of incidence rates; most especially, the saturated incidence rate.

Muhammad Altaf Khan et al. (2016) [22] studied the dynamical behavior of a Leptospirosis disease with saturated incidence rate. The model population was divided into 2 main classes viz: 
the human and the vectors having 3 and 2 compartments respectively; the susceptible, infected, recovered/removed for the humans; susceptible and infected for the rodents.

The dynamical behavior was investigated and the numerical shows that the presence or introduction of a saturation parameter declines the population in all the compartments causing the system to approach a DFE.

Jinhong Zhang et al. [23] studied an SEIR epidemic model with saturated incidence and saturated treatment function by modifying an existing SEIR model.

Their numerical simulation results revealed clearly, the advantage of saturated incidence function over the bilinear and standard incidence rates. Similarly as in [24-25]

\subsection{MODEL FORMULATION}

In this model, we have subdivided the total ' $N$ ' population into two main groups:

1. $\mathbf{N}_{\mathbf{H}}-$ Total Human population

2. $\mathrm{N}_{\mathrm{R}}-$ Total Rodent population

And from the subdivided populations $\left(N_{H}\right.$ and $\left.N_{R}\right)$, there are four (4) and three (3) compartments (sub-populations) respectively.

We have for $\mathrm{N}_{\mathrm{H}}$; the Susceptible Humans, Exposed humans, Infected humans and recovered Humans:

$$
N_{H}=S_{H}+E_{H}+I_{H}+R_{H}
$$

Also, for $\mathrm{N}_{\mathrm{R}}$; the Susceptible Rodents, Exposed Rodents and Infected Rodents:

$$
N_{R}=S_{R}+E_{R}+I_{R}
$$

This implies that:

$$
\begin{aligned}
& N=N_{H}+N_{R} \\
& N_{H}=S_{H}+E_{H}+I_{H}+R_{H} \\
& N_{R}=S_{R}+E_{R}+I_{R} \\
& \therefore N=S_{H}+E_{H}+I_{H}+R_{H}+S_{R}+E_{R}+I_{R}
\end{aligned}
$$

\subsection{MODEL ASSUMPTIONS}

Major Assumptions of the model: 
1. The Humans are made up of 4 compartments $S_{H}, E_{H}, I_{H}$ and $R_{H}$, such that $\mathrm{N}_{\mathrm{H}} \in R_{+}^{4}$.

2. The Rodents are made up of 3 compartments $S_{R}, E_{R}$ and $I_{R}$, such that $\mathrm{N}_{\mathrm{R}} \in R_{+}^{3}$.

3. Humans and Rodents get recruited into the system at the rates $\Omega_{H}$ and $\Omega_{R}$ respectively.

4. The Humans and Rodents get infected at a nonlinear incidence (saturated) rate of $\Psi_{1}$ and $\Psi_{2}$, which is also the forces of infection respectively

5. Any human can die naturally at the rate $\mu_{H}$ and due to the disease at a rate $\delta$.

6. Infected Humans gets treated at a rate $\eta$

7. The susceptible rodents (rattus-rattus or the young mastomys) come in contact with the wild rodents (Mastomys natalensis) and due to exposure, causing them to move to the exposed compartment.

8. The infected rodents do not recover for life while the susceptible human can lose immunity after recovery causing the humans re-susceptibility to the lassa virus infection.

\subsection{THE MODEL COMPARTMENTS}

The susceptible human compartment is generated by recruited humans at a rate $\Omega_{H}$ moving into being susceptible, decreased by the contact of the Lassa fever infection at a saturated incidence rate $\Psi_{1}$ (force of infection), natural death of humans at a rate $\mu_{H}$ as well.

Later, this compartment increases due to humans who lost their immunity at a rate $\rho$ after recovering.

Hence, the rate of change of the susceptible human compartment is given by:

$$
\frac{d S_{H}}{d t}=\Omega_{H}-\Psi_{1} S_{H}-\mu S_{H}+\rho R_{H}
$$

$\Psi_{1}$ is the saturated incidence function or force of infection for the humans given by:

$$
\Psi_{1}=\left(\frac{\beta_{1} I_{H}}{I+\phi I_{H}}+\frac{\beta_{2} I_{R}}{I+\phi I_{R}}\right)
$$

$\beta_{1}=$ effective contact rate capable of leading to infection

$\beta_{2}=$ effective contact rate capable of leading to infection 


$$
\frac{d S_{H}}{d t}=\Omega_{H}-\frac{\beta_{1} I_{H} S_{H}}{I+\phi I_{H}}-\frac{\beta_{2} I_{R} S_{H}}{I+\phi I_{R}}-\mu_{H} S_{H}+\rho R_{H}
$$

Where $\phi$ is the saturation parameter

The Exposed compartment is generated by humans who have acquired infection at the rate $\Psi_{1}$, and decreased by humans who die by natural cause $\mu_{H}$ and those whose infection has developed to being infectious at a rate $\alpha_{1}$.

We have the rate of change of Exposed Humans as:

$$
\begin{aligned}
& \frac{d E_{H}}{d t}=\Psi_{1} S_{H}-\alpha_{1} E_{H}-\mu_{H} E_{H} \\
& \frac{d E_{H}}{d t}=\left(\frac{\beta_{1} I_{H}}{I+\phi I_{H}}+\frac{\beta_{2} I_{R}}{I+\phi I_{R}}\right) S_{H}-\left(\alpha_{1}+\mu_{H}\right) E_{H}
\end{aligned}
$$

Where $\phi$ is the saturation parameter

This compartment is generated by those moved from being exposed to being infectious at a rate $\alpha_{1}$. The compartment is the decreased humans who die due to the disease at a rate $\delta$, by natural cause $\mu_{H}$ and those who are recovering from the disease when treated at a rate $\eta$. Hence, the rate of change of the infected compartment is given by:

$$
\frac{d I_{H}}{d t}=\alpha_{1} E_{H}-\left(\mu_{H}+\eta+\delta\right) I_{H}
$$

The Recovered compartment is generated by those who are recovering at a rate $\eta$ as a result of treatment and this compartment is reduced or decreased by humans who die due to natural cause $\mu_{H}$ and those who lose their immunity and become susceptible again at a rate $\rho$. Hence, the rate of change of the Recovered compartment is

$$
\frac{d R_{H}}{d t}=\eta I_{H}-\left(\mu_{H}+\rho\right) R_{H}
$$

The susceptible rodents' compartment is generated by rodents that gets recruited into the compartment by birth or immigration at a rate $\Omega_{R}$.

The compartment decreases by the contact of the infection from an infected human or rodent at a rate $\Psi_{2}$ which is the force of infection, and by natural death $\mu_{H}$. 


$$
\begin{gathered}
\Psi_{2}=\frac{\beta_{3} I_{R}}{1+\phi I_{R}} \\
\therefore \frac{d S_{R}}{d t}=\Omega_{R}-\Psi_{2} S_{R}-\mu_{R} S_{R}
\end{gathered}
$$

The Exposed Rodents compartment is generated by those that gets infected at a rate of $\Psi_{2}$ and decreases by Rodents that die by natural cause $\mu_{H}$ and those rodents that progresses from being exposed to being infectious at a rate $\alpha_{2}$.

The rate of change of the Exposed Rodents is given by:

$$
\begin{aligned}
& \frac{d E_{R}}{d t}=\Psi_{2} S_{R}-\alpha_{2} E_{R}-\mu_{R} E_{R} \\
& \frac{d E_{R}}{d t}=\Psi_{2} S_{R}-\left(\alpha_{2}+\mu_{R}\right) E_{R}
\end{aligned}
$$

This compartment gets increased by those that progresses from being exposed to being infected $\alpha_{2}$, and those who are being hunted or naturally died at a rate $\mu_{R}$.

The rate of change is given by:

$$
\frac{d I_{R}}{d t}=\alpha_{2} E_{R}-\mu_{R} I_{R}
$$

The Flow diagram of the model is given as:

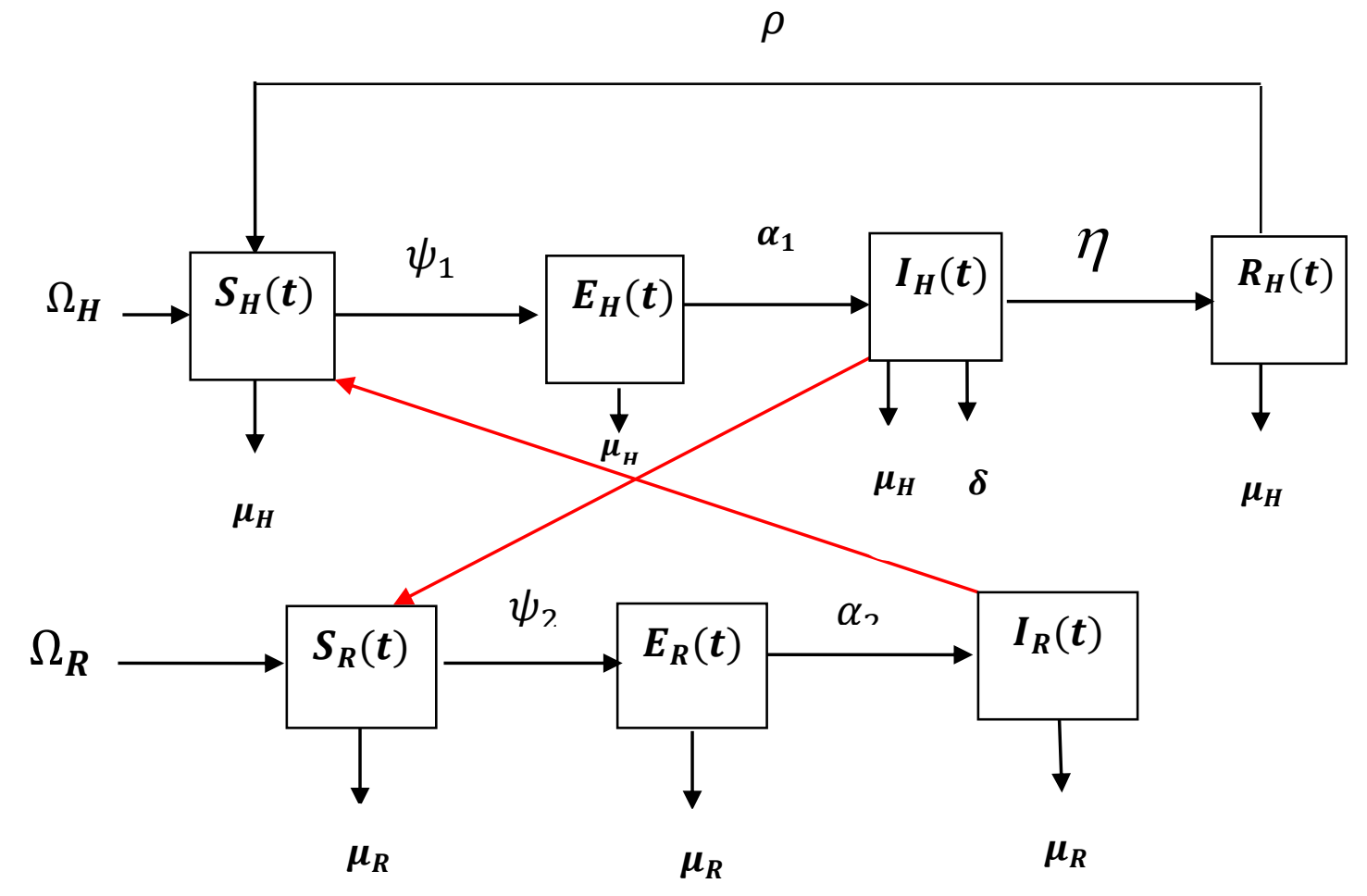


The 7-dimensional nonlinear ordinary differential equation of this model is given by:

$$
\begin{aligned}
\frac{d S_{H}}{d t} & =\Omega_{H}-\Psi_{1} S_{H}-\mu_{H} S_{H}+\rho R_{H} \\
\frac{d E_{H}}{d t} & =\Psi_{1} S_{H}-\left(\alpha_{1}+\mu_{H}\right) E_{H} \\
\frac{d I_{H}}{d t} & =\alpha_{1} E_{H}-\left(\delta+\mu_{H}+\eta\right) I_{H} \\
\frac{d R_{H}}{d t} & =\eta I_{H}-\left(\mu_{H}+\rho\right) R_{H} \\
\frac{d S_{R}}{d t} & =\Omega_{R}-\Psi_{2} S_{R}-\mu_{R} S_{R} \\
\frac{d E_{R}}{d t} & =\Psi_{2} S_{R}-\left(\alpha_{2}+\mu_{R}\right) E_{R} \\
\frac{d I_{R}}{d t} & =\alpha_{2} E_{R}-\mu_{R} I_{R}
\end{aligned}
$$

The Forces of infection of the Humans' and the rodents' is given by:

$$
\begin{aligned}
& \Psi_{1}=\frac{\beta_{1} I_{H}}{1+\phi I_{H}}+\frac{\beta_{2} I_{H}}{1+\phi I_{H}} ; \\
& \Psi_{2}=\frac{\beta_{3} I_{R}}{1+\phi I_{R}}
\end{aligned}
$$

$\beta_{1}$ and $\beta_{2}$ are effective contact rates leading to an infection for the Human and Rodent respectively while $\phi$ is the saturation parameter.

\subsubsection{MODEL STATE VARIABLES AND PARAMETERS}

Table 1

\section{STATE DESCRIPTION}

\section{VARIABLES}

\begin{tabular}{ll}
\hline$S_{H}(t)$ & The Susceptible Human \\
$E_{H}(t)$ & The Exposed Human \\
$I_{H}(t)$ & The Infected Human \\
$R_{H}(t)$ & The Recovered Human
\end{tabular}




$\begin{array}{ll}S_{R}(t) & \text { The Susceptible Rodent } \\ E_{R}(t) & \text { The Exposed Rodent } \\ I_{R}(t) & \text { The Infected Rodent }\end{array}$

\begin{tabular}{ll}
\hline PARAMETER & DESCRIPTION \\
\hline$\Omega_{H}$ & Recruitment rate of Humans \\
$\Omega_{R}$ & Recruitment rate of Rodents \\
$\beta_{1}$ & Effective contact rate between humans and mastomys rat \\
$\beta_{2}$ & Effective contact rate for susceptible rodents and wild \\
& rodents (mastomys) \\
$\beta_{3}$ & Effective contact rate leading to infection for the rodents \\
$\alpha_{1}$ & Progression rate from exposed to infectious for Humans \\
$\alpha_{2}$ & Progression rate from exposed to infectious for Rodents \\
$\Psi_{1}$ & Saturation function for Humans (Force of Infection) \\
$\Psi_{2}$ & Saturation function for Rodents (Force of Infection) \\
$\eta$ & Recovery rate for Humans \\
$\mu_{H}$ & Natural death rate of Humans \\
$\mu_{R}$ & Natural death/Hunting rate of Rodents \\
$\delta$ & Disease induced death for Humans \\
$\rho$ & Loss of immunity for humans/Rate of being Re-susceptible \\
\hline
\end{tabular}

\subsection{MATHEMATICAL ANALYSIS OF THE MODEL}

\subsubsection{POSITIVITY AND BOUNDEDNESS OF SOLUTION}

Here, we check and validate if the model is mathematically and epidemically well-posed. Also, that the solutions of the respective compartments are non-negative. We have the total Human population $N_{H}=S_{H}+E_{H}+I_{H}+R_{H}$ and the total Rodent population as $N_{R}=S_{R}+E_{R}+I_{R}$

$$
\begin{aligned}
& \therefore N_{H}=S_{H}+E_{H}+I_{H}+R_{H} \\
& N_{R}=S_{R}+E_{R}+I_{R}
\end{aligned}
$$

From the derivatives of sums we have; 


$$
\frac{d N_{H}}{d t}=\frac{d S_{H}}{d t}+\frac{d E_{H}}{d t}+\frac{d I_{H}}{d t}+\frac{d R_{H}}{d t}
$$

This implies that

$$
\begin{gathered}
\frac{d N_{H}}{d t}=\left(\Omega_{H}-\Psi_{1} S_{H}-\mu_{H} S_{H}+\rho R_{H}\right)+\left(\Psi_{1} S_{H}-\alpha_{1} E_{H}-\mu_{H} E_{H}\right) \\
+\left(\alpha_{1} E_{H}-\delta I_{H}-\mu_{H} I_{H}-\eta I_{H}\right)+\left(\eta I_{H}-\mu_{H} R_{H}-\rho R_{H}\right) \\
\therefore \frac{d N_{H}}{d t}=\Omega_{H}-\mu_{H} S_{H}-\mu_{H} E_{H}-\delta I_{H}-\mu_{H} I_{H}-\mu_{H} R_{H}
\end{gathered}
$$

Thus, we can write that;

$$
\frac{d N_{H}}{d t} \leq \Omega_{H}-\mu_{H} N_{H}
$$

We now solve the equation (19) using the integrating factor method in order to arrive at the following inequality;

$$
\begin{aligned}
& N_{H} \leq \frac{\Omega_{H}}{\mu_{H}}+C e^{-\mu_{H} t} \\
& \therefore \lim _{t \rightarrow \infty} N_{H}(t) \leq \frac{\Omega_{H}}{\mu_{H}}
\end{aligned}
$$

From (21) above, at infinite time $t=\infty$ we can clearly see that the entire population does not go into extinction. Also the result in (21) above denotes the "carrying capacity" of the system, showing that $N_{H}(t)$ is bounded as required.

Let the region of Humans be $\Gamma_{H}$; the equation (21) verifies that

$$
\Gamma_{H} \subset R_{+}^{4}
$$

Where,

$$
\Gamma_{H}=\left\{\left(S_{H}, E_{H}, I_{H}, R_{H}\right) \in R_{+}^{4}: S_{H}+E_{H}+I_{H}+R_{H} \leq \frac{\Omega_{H}}{\mu_{H}}, S_{H}>0, E_{H} \geq 0, I_{H} \geq 0, R_{H} \geq 0\right\}
$$

We proceed again to verify for the Rodent system of equations. We have the total $N$ population of the Rodents to be $N_{R}=S_{R}+E_{R}+I_{R}$;

From Cauchy's differential theorem

$$
\frac{d N_{R}(t)}{d t}=\frac{\partial N_{R}}{\partial S_{R}} \cdot \frac{d S_{R}}{d t}+\frac{\partial N_{R}}{\partial E_{R}} \cdot \frac{d E_{R}}{d t}+\frac{\partial N_{R}}{\partial I_{R}} \cdot \frac{d I_{R}}{d t}
$$




$$
\begin{gathered}
\frac{\partial N_{R}(t)}{\partial S_{R}}=1, \frac{\partial N_{R}(t)}{\partial E_{R}}=1, \frac{\partial N_{R}(t)}{\partial I_{R}}=1 ; \\
\therefore \frac{d N_{R}(t)}{d t}=\frac{d S_{R}}{d t}+\frac{d E_{R}}{d t}+\frac{d I_{R}}{d t} \\
\frac{d N_{R}(t)}{d t}=\left(\Omega_{H}-\Psi S_{H}-\mu_{H} S_{H}\right)+\left(\Psi S_{H}-\alpha_{2} E_{R}-\mu_{R} E_{R}\right)+\left(\alpha_{2} E_{R}-\mu_{R} I_{R}\right)
\end{gathered}
$$

We also obtain that

$$
\frac{d N_{R}(t)}{d t}=\Omega_{R}-\mu_{R} N_{R}
$$

Similarly as the Human system we solve by the integrating factor method and we also obtain;

$$
\lim _{t \rightarrow \infty} N_{R}(t)=\frac{\Omega_{R}}{\mu_{R}}
$$

The above shows the carrying capacity of the rodents system also showing that $N_{R}(t)$ is bounded as required.

Let the region of the Rodents be $\Gamma_{R}$; the equation (26) verifies that $\Gamma_{R} \subset R_{+}^{3}$, where

$$
\Gamma_{R}=\left\{\left(S_{R}, E_{R}, I_{R}\right) \in R_{+}^{3}: S_{R}+E_{R}+I_{R} \leq \frac{\Omega_{R}}{\mu_{R}}, S_{R}>0, E_{R} \geq 0, I_{R} \geq 0\right\}
$$

The above positivity and boundedness analysis which makes us verify equations (22) and (27) is the proof to the following theorem:

We deduce the theorem that:

2.3.2 THEOREM 1: The feasible region $\Gamma=\Gamma_{H} \times \Gamma_{R} \subset R_{+}^{4} \times R_{+}^{3}$ of the model given by:

$$
\Gamma=\left\{\begin{array}{l}
\left(S_{H}, E_{H}, I_{H}, R_{H}, S_{R}, E_{R}, I_{R}\right): S_{H}(t)+E_{H}(t)+I_{H}(t)+R_{H}(t) \leq \frac{\Omega_{H}}{\mu_{H}} ; \\
S_{R}(t)+E_{R}(t)+I_{R}(t) \leq \frac{\Omega_{R}}{\mu_{R}} ; S_{H}>0, E_{H} \geq 0, I_{H} \geq 0, R_{H} \geq 0, S_{R}>0, E_{R} \geq 0, I_{R} \geq 0
\end{array}\right\} \text { is positively }
$$

invariant and bounded

\subsubsection{EXISTENCE OF THE DISEASE-FREE EQUILIBRIUM POINT}

The disease-free equilibrium points are steady-state solutions where the entire population is free from Lassa haemorrhagic virus infection, i.e no Lassa haemorrhagic virus infection which implies the entire population is susceptible. 
Then, $S_{H}^{0} \neq 0, S_{V}^{0} \neq 0$

We obtain the DFE point $E^{0}$, by setting exposed class $E(t)$, Infections class $I(t)$ and removed class/recovered class $R(t)$ at zero.

Here, $E_{H}(t)=0, I_{H}(t)=0, R_{H}(t)=0, S_{R}(t)=0, E_{R}(t)=0, I_{R}(t)=0$

Automatically, this implies that their derivatives are zero including the derivative of the susceptible.

$$
\frac{d S_{H}(t)}{d t}=0, \frac{d E_{H}(t)}{d t}=0, \frac{d I_{H}(t)}{d t}=0, \frac{d R_{H}(t)}{d t}=0, \frac{d S_{R}(t)}{d t}=0, \frac{d E_{R}(t)}{d t}=0, \frac{d I_{R}(t)}{d t}=0
$$

Then, we have or new system of equation after setting all derivatives to zero as:

$$
\begin{aligned}
& 0=\Omega_{H}-\frac{\beta_{1} S_{H} I_{H}}{1+\phi I_{H}}-\frac{\beta_{2} S_{H} I_{R}}{1+\phi I_{R}}-\mu_{H} S_{H}+\rho R_{H} \\
& 0=\frac{\beta_{1} S_{H} I_{H}}{1+\phi I_{H}}+\frac{\beta_{2} S_{H} I_{R}}{1+\phi I_{R}}-\left(\alpha_{1}+\mu_{H}\right) E_{H} \\
& 0=\alpha_{1} E_{H}-\left(\mu_{H}+\delta+\eta\right) I_{H} \\
& 0=\eta I_{H}-\left(\mu_{H}+\rho\right) R_{H} \\
& 0=\Omega_{R}-\frac{\beta_{3} S_{R} I_{R}}{1+\phi I_{R}}-\mu_{R} S_{R} \\
& 0=\frac{\beta_{3} S_{R} I_{R}}{1+\phi I_{R}}-\left(\alpha_{2}+\mu_{R}\right) E_{R} \\
& 0=\alpha_{2} I_{R}-\mu_{R} I_{R}
\end{aligned}
$$

At equilibrium we have,

$$
\begin{aligned}
& \frac{d S_{H}^{0}}{d t}=0=\Omega_{H}-\frac{\beta_{1} S_{H}^{0} I_{H}^{0}}{1+\phi I_{H}^{0}}-\frac{\beta_{2} S_{H}^{0} I_{R}^{0}}{1+\phi I_{R}^{0}}-\mu_{H} S_{H}^{0}+\rho R_{H}^{0} \\
& \frac{d E_{H}^{0}}{d t}=0=\frac{\beta_{1} S_{H}^{0} I_{H}^{0}}{1+\phi I_{H}^{0}}+\frac{\beta_{2} S_{R}^{0} I_{R}^{0}}{1+\phi I_{R}^{0}}-\left(\alpha_{1}+\mu_{H}\right) E_{H}^{0} \\
& \frac{d I_{H}^{0}}{d t}=0=\alpha_{1} E_{H}^{0}-\left(\mu_{H}+\delta+\eta\right) I_{H}^{0} \\
& \frac{d R_{H}^{0}}{d t}=0=\eta I_{H}^{0}-\left(\mu_{H}+\rho\right) R_{H}^{0} \\
& \frac{d S_{R}^{0}}{d t}=0=\Omega_{R}-\frac{\beta_{3} S_{R}^{0} I_{R}^{0}}{1+\phi I_{R}^{0}}-\mu_{R}^{0} S_{R}^{0} \\
& \frac{d E_{R}^{0}}{d t}=0=\frac{\beta_{3} S_{R}^{0} I_{R}^{0}}{1+\phi I_{R}^{0}}-\left(\alpha_{2}+\mu_{R}\right) S_{R}^{0} \\
& \frac{d I_{R}^{0}}{d t}=0=\alpha_{2} E_{R}^{0}-\mu_{R} I_{R}^{0}
\end{aligned}
$$


$S_{H}^{0} \neq 0, E_{H}^{0}=0, I_{H}^{0}=0, R_{H}^{0}=0$

Thus, the system (31) now becomes

$\Omega_{H}-\mu_{H} S_{H}^{0}=0$

$\Omega_{R}-\mu_{R} S_{R}^{0}=0$

This gives

$$
S_{H}^{0}=\frac{\Omega_{H}}{\mu_{H}}, S_{R}^{0}=\frac{\Omega_{R}}{\mu_{R}}
$$

This gives us the DFE point for the Human and the Rodent population.

$$
\therefore E^{0}=\left(S_{H}^{0}, E_{H}^{0}, I_{H}^{0}, R_{H}^{0}, S_{R}^{0}, E_{R}^{0}, I_{R}^{0}\right)=\left(\frac{\Omega_{H}}{\mu_{H}}, 0,0,0, \frac{\Omega_{R}}{\mu_{R}}, 0,0\right)
$$

is the DFE point for the system.

\subsubsection{THE BASIC REPRODUCTION NUMBER $R_{0}$ OF THE HUMAN-RODENT MODEL FOR THE LASSA FEVER TRANSMISSION MODEL}

An important concept in Epidemiological models is the Basic Reproduction number $R_{0}$.

This number is defined as the average number of secondary infections in the $E(t)$ compartment, infected by an infective individual already in $I(t)$ all in a completely susceptible population.

$R_{0}$ is an important parameter that gives us the account whether an infection will spread through the population or not. To obtain $R_{0}$, we would use the "Next generation matrix method".

Now, if we have a system in which they are multiple discrete types of infected individuals (E.g. human and mosquitoes, men and women, human and chickens etc.).

We define the next generation matrix the square matrix ' $G$ ' in which $i j^{\text {th }}$ element of $G, g_{i j}$ is the expected number of secondary infections of type $i$ caused by a single infected individual of type $j$ assuming that the population of type $i$ is entirely susceptible.

The assumption that the entire population is susceptible implies that the basic reproduction number would be computed at the disease free equilibrium point $E^{0}$.

This means that each element of matrix $G$ is a reproduction number but "the one in which who infects whom is accounted for". 
Shortly speaking, the basic reproduction number of this model would be computed using the next-generation matrix as defined in Van den Driesshe \& Watmough (2002) and Diekmann, Heesterbeek \& Metz (1990) [30-31]. We denote the reproduction number of the human population (Human) by $R_{0 H}$ and the rodent population (Rats) reproduction number by $R_{0 R}$ From the human nonlinear system of ODEs;

$$
\begin{aligned}
\frac{d S_{H}}{d t} & =\Omega_{H}-\frac{\beta_{1} S_{H} I_{H}}{1+\phi I_{H}}-\frac{\beta_{2} S_{R} I_{R}}{1+\phi I_{R}}-\mu_{H} S_{H}+\rho R_{H} \\
\frac{d E_{H}}{d t} & =\frac{\beta_{1} S_{H} I_{H}}{1+\phi I_{H}}+\frac{\beta_{2} S_{R} I_{R}}{1+\phi I_{R}}-\left(\mu_{H}+\alpha_{1}\right) E_{H} \\
\frac{d I_{H}}{d t} & =\alpha_{1} E_{H}-\left(\eta+\delta+\mu_{H}\right) I_{H} \\
\frac{d R_{H}}{d t} & =\eta I_{H}-\left(\mu_{H}+\rho\right) R_{H}
\end{aligned}
$$

Using the next generation matrix method,

Let

$$
X=\left(E_{H}, I_{H}, S_{H}, R_{H}\right)^{T}
$$

Then

$$
\frac{d X_{H}}{d t}=\frac{d}{d t}\left[\begin{array}{c}
E_{H} \\
I_{H} \\
S_{H} \\
R_{H}
\end{array}\right]=\left[\begin{array}{c}
\frac{d E_{H}}{d t} \\
\frac{d I_{H}}{d t} \\
\frac{d S_{H}}{d t} \\
\frac{d R_{H}}{d t}
\end{array}\right]=\left[\begin{array}{c}
E_{H}^{\prime} \\
I_{H}^{\prime} \\
S_{H}^{\prime} \\
R_{H}^{\prime}
\end{array}\right]=\left[\begin{array}{l}
\frac{\beta_{1} S_{H} I_{H}}{1+\phi I_{H}}+\frac{\beta_{2} S_{R} I_{R}}{1+\phi I_{R}}-\left(\mu_{H}+\alpha_{1}\right) E_{H} \\
\alpha_{1} E_{H}-\left(\eta+\delta+\mu_{H}\right) I_{H} \\
\Omega_{H}-\frac{\beta_{1} S_{H} I_{H}}{1+\phi I_{H}}-\frac{\beta_{2} S_{R} I_{R}}{1+\phi I_{R}}-\mu_{H} S_{H}+\rho R_{H} \\
\alpha_{2} I_{H}-\left(\mu_{H}+\rho\right) R_{H}
\end{array}\right]
$$

By splitting the matrix in the equation (31) we have;

$$
\frac{d X_{H}}{d t}=\left[\begin{array}{c}
\frac{\beta_{1} S_{H} I_{H}}{1+\phi I_{H}}+\frac{\beta_{2} S_{R} I_{R}}{1+\phi I_{R}} \\
0 \\
0 \\
0
\end{array}\right]-\left[\begin{array}{l}
\left(\mu_{H}+\alpha_{1}\right) E_{H} \\
-\alpha_{1} E_{H}+\left(\eta+\delta+\mu_{H}\right) I_{H} \\
-\Omega_{H}+\frac{\beta_{1} S_{H} I_{H}}{1+\phi I_{H}}+\frac{\beta_{2} S_{R} I_{R}}{1+\phi I_{R}}+\mu_{H} S_{H}-\rho R_{H} \\
-\alpha_{2} I_{H}+\left(\mu_{H}+\rho\right) R_{H}
\end{array}\right]
$$


This is now in the form

$$
\begin{gathered}
\frac{d X_{H}}{d t}=F_{i}(X)-V_{i}(X) \\
F_{i}(X)=\left[\begin{array}{c}
\frac{\beta_{1} S_{H} I_{H}}{1+\phi I_{H}}+\frac{\beta_{2} S_{R} I_{R}}{1+\phi I_{R}} \\
0 \\
0 \\
0
\end{array}\right]=\left[\begin{array}{c}
F_{1} \\
F_{2} \\
F_{3} \\
F_{4}
\end{array}\right] ; V_{i}(X)=\left[\begin{array}{l}
\left(\mu_{H}+\alpha_{1}\right) E_{H} \\
-\alpha_{1} E_{H}+\left(\eta+\delta+\mu_{H}\right) I_{H} \\
-\Omega_{H}+\frac{\beta_{1} S_{H} I_{H}}{1+\phi I_{H}}+\frac{\beta_{2} S_{R} I_{R}}{1+\phi I_{R}}+\mu_{H} S_{H}-\rho R_{H} \\
-\alpha_{2} I_{H}+\left(\mu_{H}+\rho\right) R_{H}
\end{array}\right]=\left[\begin{array}{c}
V_{1} \\
V_{2} \\
V_{3} \\
V_{4}
\end{array}\right]
\end{gathered}
$$

Where $F_{i}(X)$ is the matrix of new infections and $V_{i}(X)$ is the matrix of other transfer terms [30]

The next step here is to linearize the matrix $F_{i}(X)$ and $V_{i}(X)$ by taking the jacobian of each term in the matrices at Disease free equilibrium point $E^{0}$.

Let $J\left[F_{i}(X)\right]=F_{H}$ and $J\left[V_{i}(X)\right]=V_{H}$

$$
\begin{gathered}
\therefore F_{H}=\frac{\partial F_{i}(X)}{\partial X_{j}}, V_{H}=\frac{\partial V_{i}(X)}{\partial X_{j}} \quad \text { At DFE } F_{H}\left(E^{0}\right)=\frac{\partial F_{i}\left(E^{0}\right)}{\partial X_{j}}, V_{H}\left(E^{0}\right)=\frac{\partial V_{i}\left(E^{0}\right)}{\partial X_{j}} \\
\left.\frac{\partial F_{i}\left(E^{0}\right)}{\partial X_{j}}=\left(\begin{array}{llll}
\frac{\partial F_{1}\left(E^{0}\right)}{\partial E_{H}} & \frac{\partial F_{1}\left(E^{0}\right)}{\partial I_{H}} & \frac{\partial F_{1}\left(E^{0}\right)}{\partial S_{H}} & \frac{\partial F_{1}\left(E^{0}\right)}{\partial R_{H}} \\
\frac{\partial F_{2}\left(E^{0}\right)}{\partial E_{H}} & \frac{\partial F_{2}\left(E^{0}\right)}{\partial I_{H}} & \frac{\partial F_{2}\left(E^{0}\right)}{\partial S_{H}} & \frac{\partial F_{2}\left(E^{0}\right)}{\partial R_{H}} \\
\frac{\partial F_{3}\left(E^{0}\right)}{\partial E_{H}} & \frac{\partial F_{3}\left(E^{0}\right)}{\partial I_{H}} & \frac{\partial F_{3}\left(E^{0}\right)}{\partial S_{H}} & \frac{\partial F_{3}\left(E^{0}\right)}{\partial R_{H}} \\
\frac{\partial F_{4}\left(E^{0}\right)}{\partial E_{H}} & \frac{\partial F_{4}\left(E^{0}\right)}{\partial I_{H}} & \frac{\partial F_{4}\left(E^{0}\right)}{\partial S_{H}} & \frac{\partial F_{4}\left(E^{0}\right)}{\partial R_{H}}
\end{array}\right) ; \begin{array}{llll}
\frac{\partial V_{1}\left(E^{0}\right)}{\partial E_{H}} & \frac{\partial V_{1}\left(E^{0}\right)}{\partial I_{H}} & \frac{\partial V_{1}\left(E^{0}\right)}{\partial S_{H}} & \frac{\partial V_{1}\left(E^{0}\right)}{\partial R_{H}} \\
\frac{\partial V_{2}\left(E^{0}\right)}{\partial E_{H}} & \frac{\partial V_{2}\left(E^{0}\right)}{\partial I_{H}} & \frac{\partial V_{2}\left(E^{0}\right)}{\partial S_{H}} & \frac{\partial V_{2}\left(E^{0}\right)}{\partial R_{H}} \\
\frac{\partial V_{3}\left(E^{0}\right)}{\partial E_{H}} & \frac{\partial V_{3}\left(E^{0}\right)}{\partial I_{H}} & \frac{\partial V_{3}\left(E^{0}\right)}{\partial S_{H}} & \frac{\partial V_{3}\left(E^{0}\right)}{\partial R_{H}} \\
\frac{\partial V_{4}\left(E^{0}\right)}{\partial E_{H}} & \frac{\partial V_{4}\left(E^{0}\right)}{\partial I_{H}} & \frac{\partial V_{4}\left(E^{0}\right)}{\partial S_{H}} & \frac{\partial V_{4}\left(E^{0}\right)}{\partial R_{H}}
\end{array}\right)
\end{gathered}
$$

For the Reproduction number, we only need terms in the Exposed and the infected compartments [27].

Then we have the matrix

$$
\operatorname{NewF}\left(E^{0}\right)=F_{H}=\left[\begin{array}{cc}
0 & \beta_{1} \frac{\Omega_{H}}{\mu_{H}} \\
0 & 0
\end{array}\right] \text { And } N e w V\left(E^{0}\right)=V_{H}=\left[\begin{array}{cc}
\left(\mu_{H}+\alpha_{1}\right) & 0 \\
-\alpha_{1} & \left(\mu_{H}+\delta+\eta\right)
\end{array}\right]
$$


$R_{0 H}$ Is the spectral radius or dominant Eigen value of $\left(F_{H} V_{H}^{-1}\right)$ that is the eigenvalue of $\left|\left(F_{H} V_{H}^{-1}\right)-\lambda I\right|=0 \mathrm{I}$ is an identity matrix.

$$
F V^{-1}=\left[\begin{array}{cc}
\frac{\alpha_{1} \beta_{1} \Omega_{H}}{\mu_{H}\left(\mu_{H}+\alpha_{1}\right)\left(\mu_{H}+\delta+\eta\right)} & \frac{\beta_{1} \Omega_{H}}{\mu_{H}\left(\mu_{H}+\delta+\eta\right)} \\
0 & 0
\end{array}\right]
$$

We now seek the spectral radius of $F V^{-1}$

$$
\left|F V^{-1}-\lambda I\right|=\llbracket\left[\begin{array}{cc}
\frac{\alpha_{1} \beta_{1} \Omega_{H}}{\mu_{H}\left(\mu_{H}+\alpha_{1}\right)\left(\mu_{H}+\delta+\eta\right)}-\lambda & \frac{\beta_{1} \Omega_{H}}{\mu_{H}\left(\mu_{H}+\delta+\eta\right)} \\
0 & 0-\lambda
\end{array}\right] \mid=0
$$

By computing the spectral radius of (35), the reproduction number is given as;

$$
R_{0 H}=\frac{\alpha_{1} \beta_{1} \Omega_{H}}{\mu_{H}\left(\mu_{H}+\alpha_{1}\right)\left(\mu_{H}+\delta+\eta\right)}
$$

Let $\left(\alpha_{1}+\mu_{H}\right)=X ;\left(\mu_{H}+\delta+\eta\right)=U$

$$
\therefore R_{0 H}=\frac{\alpha_{1} \beta_{1} \Omega_{H}}{\mu_{H} X U}
$$

Similarly, by considering the nonlinear system in the Rodents' model

$$
\begin{aligned}
\frac{d S_{R}}{d t} & =\Omega_{R}-\frac{\beta_{3} S_{R} I_{R}}{1+\phi I_{R}}-\mu_{R} S_{R} \\
\frac{d E_{R}}{d t} & =\frac{\beta_{3} S_{R} I_{R}}{1+\phi I_{R}}-\left(\alpha_{2}+\mu_{R}\right) E_{R} \\
\frac{d I_{R}}{d t} & =\alpha_{2} E_{R}-\mu_{R} I_{R}
\end{aligned}
$$

Similarly, using the Next generation matrix approach on the rodents system of equations above we have the Rodents' reproduction number as

$$
R_{0 R}=\frac{\alpha_{2} \beta_{3} \Omega_{R}}{\mu_{R}^{2}\left(\alpha_{2}+\mu_{R}\right)}
$$

The reproduction number of a standard Rodent-Human interactive according to [30-31] model is given by

$$
R_{G}=\sqrt{R_{0 H} R_{0 R}}
$$




$$
R_{G}=\sqrt{\frac{\alpha_{1} \alpha_{2} \beta_{1} \beta_{3} \Omega_{H} \Omega_{R}}{\mu_{H} \mu_{R}^{2}\left(\alpha_{1}+\mu_{H}\right)\left(\mu_{H}+\delta+\eta\right)\left(\alpha_{2}+\mu_{R}\right)}}
$$

Let

$$
\begin{aligned}
& \left(\alpha_{1}+\mu_{H}\right)=X \\
& \left(\alpha_{2}+\mu_{R}\right)=U \\
& \left(\mu_{H}+\delta+\eta\right)=Y
\end{aligned} ;
$$

$$
\begin{gathered}
R_{G}=\sqrt{\frac{\alpha_{1} \alpha_{2} \beta_{1} \beta_{3} \Omega_{H} \Omega_{R}}{\mu_{H} \mu_{R}^{2} X Y U}} \\
\therefore R_{G}^{2}=R_{0 H} R_{0 R}
\end{gathered}
$$

\subsubsection{STABILITY ANALYSIS OF THE DISEASE-FREE EQUILIBRIUM}

We have already established that a disease-free equilibrium exists for the model and we have also established the carrying capacity of this model. We would now convey stability analyses on the disease-free equilibrium in order to presume the long term effect of this equilibrium as regards being stable or not.

\subsubsection{THEOREM 2:}

The DFE $E_{0 H R}\left(\frac{\Omega_{H}}{\mu_{H}}, 0,0,0, \frac{\Omega_{R}}{\mu_{R}}, 0,0\right)$ of the system is asymptotically stable if all eigenvalues of the system's jacobian are negative real values.

\section{Proof:}

To prove the above theorem, we compute the Jacobian matrix of the model's system in equation (14) at DFE point $E_{0 H R}\left(\frac{\Omega_{H}}{\mu_{H}}, 0,0,0, \frac{\Omega_{R}}{\mu_{R}}, 0,0\right)$. Then compute/evaluate the eigenvalues of the system.

We have the Jacobian matrix of the model to be given as: 


$$
J=\left[\begin{array}{ccccccc}
-\Psi_{1}-\mu_{H} & 0 & \frac{\beta_{1} S_{H}}{\left(1+\phi I_{H}\right)^{2}} & \rho & 0 & 0 & -\frac{\beta_{2} S_{H}}{\left(1+\phi I_{R}\right)^{2}} \\
\frac{\beta_{1} I_{H}}{1+\phi I_{H}} & -\left(\alpha_{2}+\mu_{H}\right) & \frac{\beta_{1} S_{H}}{\left(1+\phi I_{H}\right)^{2}} & 0 & 0 & 0 & \frac{\beta_{2} S_{H}}{\left(1+\phi I_{R}\right)^{2}} \\
0 & \alpha_{1} & -\left(\mu_{H}+\delta+\eta\right) & 0 & 0 & 0 & 0 \\
0 & 0 & \eta & -\left(\mu_{H}+\rho\right) & 0 & 0 & 0 \\
0 & 0 & 0 & 0 & \left(-\frac{\beta_{2} I_{R}}{1+\phi I_{R}}-\mu_{R}\right) & 0 & -\frac{\beta_{3} S_{R}}{\left(1+\phi I_{R}\right)^{2}} \\
0 & 0 & 0 & 0 & \frac{\beta_{3} I_{R}}{1+\phi I_{R}} & -\left(\alpha_{2}+\mu_{R}\right) & \frac{\beta_{3} S_{R}}{\left(1+\phi I_{R}\right)^{2}} \\
0 & 0 & 0 & 0 & 0 & \alpha_{2} & \mu_{R}
\end{array}\right]
$$

Now at DFE,

$$
J=\left[\begin{array}{ccccccc}
-\mu_{H} & 0 & -\beta_{1} \frac{\Omega_{H}}{\mu_{H}} & \rho & 0 & 0 & -\beta_{2} \frac{\Omega_{H}}{\mu_{H}} \\
0 & -\left(\alpha_{2}+\mu_{H}\right) & \beta_{1} \frac{\Omega_{H}}{\mu_{H}} & 0 & 0 & 0 & \beta_{2} \frac{\Omega_{H}}{\mu_{H}} \\
0 & \alpha_{1} & -\left(\mu_{H}+\delta+\eta\right) & 0 & 0 & 0 & 0 \\
0 & 0 & \eta & -\left(\mu_{H}+\rho\right) & 0 & 0 & 0 \\
0 & 0 & 0 & 0 & -\mu_{R} & 0 & -\beta_{3} \frac{\Omega_{R}}{\mu_{R}} \\
0 & 0 & 0 & 0 & 0 & -\left(\alpha_{2}+\mu_{R}\right) & \beta_{3} \frac{\Omega_{R}}{\mu_{R}} \\
0 & 0 & 0 & 0 & 0 & \alpha_{2} & -\mu_{R}
\end{array}\right]
$$

Let $\left(\alpha_{1}+\mu_{H}\right)=X,\left(\mu_{H}+\delta+\eta\right)=Y,\left(\mu_{H}+\rho\right)=Z,\left(\alpha_{1}+\mu_{R}\right)=U$; Then;

$$
J=\left[\begin{array}{ccccccc}
-\mu_{H} & 0 & -\beta_{1} \frac{\Omega_{H}}{\mu_{H}} & \ell & 0 & 0 & -\beta_{2} \frac{\Omega_{H}}{\mu_{H}} \\
0 & -X & \beta_{1} \frac{\Omega_{H}}{\mu_{H}} & 0 & 0 & 0 & \beta_{2} \frac{\Omega_{H}}{\mu_{H}} \\
0 & \alpha_{1} & -Y & 0 & 0 & 0 & 0 \\
0 & 0 & \eta & -Z & 0 & 0 & 0 \\
0 & 0 & 0 & 0 & -\mu_{R} & 0 & -\beta_{3} \frac{\Omega_{R}}{\mu_{R}} \\
0 & 0 & 0 & 0 & 0 & -U & \beta_{3} \frac{\Omega_{R}}{\mu_{R}} \\
0 & 0 & 0 & 0 & 0 & \alpha_{2} & -\mu_{R}
\end{array}\right]
$$

Now for the eigen value computation, we have; 


$$
|J-\lambda I|=\left[\begin{array}{ccccccc}
-\mu_{H}-\lambda & 0 & -\beta_{1} \frac{\Omega_{H}}{\mu_{H}} & \rho & 0 & 0 & -\beta_{2} \frac{\Omega_{H}}{\mu_{H}} \\
0 & -X-\lambda & \beta_{1} \frac{\Omega_{H}}{\mu_{H}} & 0 & 0 & 0 & \beta_{2} \frac{\Omega_{H}}{\mu_{H}} \\
0 & \alpha_{1} & -Y-\lambda & 0 & 0 & 0 & 0 \\
0 & 0 & \eta & -Z-\lambda & 0 & 0 & 0 \\
0 & 0 & 0 & 0 & -\mu_{R}-\lambda & 0 & -\beta_{3} \frac{\Omega_{R}}{\mu_{R}} \\
0 & 0 & 0 & 0 & 0 & -U-\lambda & \beta_{3} \frac{\Omega_{R}}{\mu_{R}} \\
0 & 0 & 0 & 0 & 0 & \alpha_{2} & -\mu_{R}-\lambda
\end{array}\right]
$$

From the above matrix in equation (41), $-\mu_{H}-\lambda$ is the only non-zero-entry in the first column; Hence $\lambda_{1}=-\mu_{H}$, while we delete the perpendicular rows and columns the element falls in. We now have a new $J_{0}$ matrix as

$$
J_{0}=\left[\begin{array}{cccccc}
-X-\lambda & \beta_{1} \frac{\Omega_{H}}{\mu_{H}} & 0 & 0 & 0 & \beta_{2} \frac{\Omega_{H}}{\mu_{H}} \\
\alpha_{1} & -Y-\lambda & 0 & 0 & 0 & 0 \\
0 & \eta & -Z-\lambda & 0 & 0 & 0 \\
0 & 0 & 0 & -\mu_{R}-\lambda & 0 & -\beta_{3} \frac{\Omega_{R}}{\mu_{R}} \\
0 & 0 & 0 & 0 & -U-\lambda & \beta_{3} \frac{\Omega_{R}}{\mu_{R}} \\
0 & 0 & 0 & 0 & \alpha_{2} & -\mu_{R}-\lambda
\end{array}\right]
$$

Similarly on row 4 in equation (42), we have $-\mu_{R}-\lambda$ as the only entry. Hence we have new matrix $J_{1}$ with $\lambda_{2}=-\mu_{R}$

$$
J_{1}=\left[\begin{array}{ccccc}
-X-\lambda & \beta_{1} \frac{\Omega_{H}}{\mu_{H}} & 0 & 0 & -\beta_{3} \frac{\Omega_{R}}{\mu_{R}} \\
\alpha_{1} & -Y-\lambda & 0 & 0 & 0 \\
0 & \eta & -Z-\lambda & 0 & 0 \\
0 & 0 & 0 & -U-\lambda & -\beta_{3} \frac{\Omega_{R}}{\mu_{R}} \\
0 & 0 & 0 & \alpha_{2} & -\mu_{R}-\lambda
\end{array}\right]
$$

Similarly in row $3, \lambda_{3}=-Z$ but $Z=\left(\mu_{H}+\rho\right)$, then $\lambda_{3}=-\left(\mu_{H}+\rho\right)$

We then have a new matrix $J_{2}$ such that 


$$
J_{2}=\left[\begin{array}{cccc}
-X-\lambda & \beta_{1} \frac{\Omega_{H}}{\mu_{H}} & 0 & \beta_{2} \frac{\Omega_{H}}{\mu_{H}} \\
\alpha_{1} & -Y-\lambda & 0 & 0 \\
0 & 0 & -Z-\lambda & \beta_{3} \frac{\Omega_{R}}{\mu_{R}} \\
0 & 0 & \alpha_{2} & -\mu_{R}-\lambda
\end{array}\right]
$$

By splitting the matrix $J_{2}$ in equation (44), we have two matrices $J_{2}^{*}$ and $I \lambda$ respectively

$$
J_{2}^{*}=\left[\begin{array}{cccc}
-X & \beta_{1} \frac{\Omega_{H}}{\mu_{H}} & 0 & \beta_{2} \frac{\Omega_{H}}{\mu_{H}} \\
\alpha_{1} & -Y & 0 & 0 \\
0 & 0 & -Z & \beta_{3} \frac{\Omega_{R}}{\mu_{R}} \\
0 & 0 & \alpha_{2} & -\mu_{R}
\end{array}\right]
$$

And

$$
I \lambda=\left[\begin{array}{llll}
\lambda & 0 & 0 & 0 \\
0 & \lambda & 0 & 0 \\
0 & 0 & \lambda & 0 \\
0 & 0 & 0 & \lambda
\end{array}\right]
$$

We then perform the "Gaussian elimination method" on the matrix $J_{2}^{*}$ in equation (45), so as to reduce the matrix and solve for our eigen values. We have;

$$
\begin{aligned}
& R_{2}^{*}=R_{2}+\frac{\alpha_{1}}{X} R_{1} \\
& R_{2}^{*}=\left[\begin{array}{llll}
0 & \frac{-X Y \mu_{H}+\alpha_{1} \beta_{1} \Omega_{H}}{\mu_{H}} & 0 & \frac{\alpha_{1} \beta_{2} \Omega_{H}}{X \mu_{H}}
\end{array}\right] \\
& R_{4}^{*}=R_{4}+\frac{\alpha_{2}}{U} R_{3} \\
& R_{4}^{*}=\left[\begin{array}{llll}
0 & 0 & 0 & -\left(\mu_{R}-\frac{\alpha_{2} \beta_{3} \Omega_{R}}{\mu_{R}}\right)
\end{array}\right]
\end{aligned}
$$

Then the new reduced matrix $R_{e}\left(J_{2}^{*}\right)$ gives 


$$
R_{e}\left(J_{2}^{*}\right)=\left[\begin{array}{cccc}
-X & \beta_{1} \frac{\Omega_{H}}{\mu_{H}} & 0 & \beta_{2} \frac{\Omega_{H}}{\mu_{H}} \\
0 & -\left(X Y-\frac{\alpha_{1} \beta_{1} \Omega_{H}}{\mu_{H}}\right) & 0 & \frac{\alpha_{1} \beta_{2} \Omega_{H}}{X \mu_{H}} \\
0 & 0 & -U & \beta_{3} \frac{\Omega_{R}}{\mu_{R}} \\
0 & 0 & 0 & -\left(\mu_{R}-\frac{\alpha_{2} \beta_{3} \Omega_{R}}{\mu_{R}}\right)
\end{array}\right]
$$

For the eigenvalues we have,

$$
\left|R_{e}\left(J_{2}^{*}\right)-\lambda I\right|=\left[\begin{array}{cccc}
-X-\lambda & \beta_{1} \frac{\Omega_{H}}{\mu_{H}} & 0 & \beta_{2} \frac{\Omega_{H}}{\mu_{H}} \\
0 & -\left(X Y-\frac{\alpha_{1} \beta_{1} \Omega_{H}}{\mu_{H}}\right)-\lambda & 0 & \frac{\alpha_{1} \beta_{2} \Omega_{H}}{X \mu_{H}} \\
0 & 0 & -U-\lambda & \beta_{3} \frac{\Omega_{R}}{\mu_{R}} \\
0 & 0 & 0 & -\left(\mu_{R}-\frac{\alpha_{2} \beta_{3} \Omega_{R}}{\mu_{R}}\right)-\lambda
\end{array}\right]
$$

From equation (47), it is easy for us to implement the same matrix algorithm performed on (41); Hence we have the remaining eigenvalues as;

$$
\lambda_{5}=-\left(X Y-\frac{\alpha_{1} \beta_{1} \Omega_{H}}{\mu_{H}}\right) ; \lambda_{6}=-U ; \lambda_{7}=-\left(\mu_{R}-\frac{\alpha_{2} \beta_{3} \Omega_{R}}{\mu_{R}}\right)
$$

We then have the seven eigenvalues of the 7-dimensional system as;

$$
\begin{aligned}
& \lambda_{1}=-\mu_{h} ; \lambda_{2}=-\mu_{R} ; \lambda_{3}=-\left(\mu_{H}+\rho\right) ; \lambda_{4}=-X \\
& \lambda_{5}=-\left(X Y-\frac{\alpha_{1} \beta_{1} \Omega_{H}}{\mu_{H}}\right) ; \lambda_{6}=-U ; \lambda_{7}=-\left(\mu_{R}-\frac{\alpha_{2} \beta_{3} \Omega_{R}}{U \mu_{R}}\right)
\end{aligned}
$$

If we replace

$$
\begin{aligned}
& X=\left(\alpha_{1}+\mu_{H}\right) \\
& Y=\left(\mu_{H}+\delta+\eta\right) ; \\
& U=\left(\alpha_{2}+\mu_{R}\right)
\end{aligned}
$$

We have;

$$
\begin{aligned}
& \lambda_{1}=-\mu_{h} ; \lambda_{2}=-\mu_{R} ; \lambda_{3}=-\left(\mu_{H}+\rho\right) ; \lambda_{4}=-\left(\alpha_{1}+\mu_{H}\right) \\
& \lambda_{5}=-\left(\frac{\left(\alpha_{1}+\mu_{H}\right)\left(\mu_{H}+\delta+\eta\right) \mu_{H}-\alpha_{1} \beta_{1} \Omega_{H}}{\mu_{H}}\right) ; \lambda_{6}=-\left(\alpha_{2}+\mu_{R}\right) ; \lambda_{7}=-\left(\frac{\left(\alpha_{2}+\mu_{R}\right) \mu_{R}^{2}-\alpha_{2} \beta_{3} \Omega_{R}}{\left(\alpha_{2}+\mu_{R}\right) \mu_{R}}\right)
\end{aligned}
$$


Clearly, it is seen that all the Eigenvalues of the seven dimensional systems are negative values. This implies that the disease free equilibrium is stable.

\subsubsection{THEOREM 3}

The Disease-Free equilibrium is locally asymptotically stable when $R_{G}<1$.

\section{Proof:}

From the $\lambda_{5}$ and $\lambda_{7}$ of the eigen values obtained, we have;

$$
\begin{aligned}
& \lambda_{5}=-\left(\frac{\left(\alpha_{1}+\mu_{H}\right)\left(\mu_{H}+\delta+\eta\right) \mu_{H}-\alpha_{1} \beta_{1} \Omega_{H}}{\mu_{H}}\right) \\
& \lambda_{7}=-\left(\frac{\left(\alpha_{2}+\mu_{R}\right) \mu_{R}^{2}-\alpha_{2} \beta_{3} \Omega_{R}}{\left(\alpha_{2}+\mu_{R}\right) \mu_{R}}\right)
\end{aligned}
$$

From the eigen values above, these eigen values can be factored as:

$$
\begin{gathered}
\lambda_{5}=-\left(\alpha_{1}+\mu_{H}\right)\left(\mu_{H}+\delta+\eta\right)\left(1-\frac{\alpha_{1} \beta_{1} \Omega_{H}}{\mu_{H}\left(\alpha_{1}+\mu_{H}\right)\left(\mu_{H}+\delta+\eta\right)}\right) \\
\lambda_{7}=-\mu_{R}\left(1-\frac{\alpha_{2} \beta_{3} \Omega_{R}}{\mu_{R}^{2}\left(\alpha_{2}+\mu_{R}\right)}\right)
\end{gathered}
$$

From the above, we have can write that;

$$
\begin{gathered}
\lambda_{5}=-\left(\alpha_{1}+\mu_{H}\right)\left(\mu_{H}+\delta+\eta\right)\left(1-\frac{\alpha_{1} \beta_{1} \Omega_{H}}{\mu_{H}\left(\alpha_{1}+\mu_{H}\right)\left(\mu_{H}+\delta+\eta\right)}\right) \\
\leq-\left(\alpha_{1}+\mu_{H}\right)\left(\mu_{H}+\delta+\eta\right)\left(1-R_{0 H}\right) \\
\lambda_{7}=-\mu_{R}\left(1-\frac{\alpha_{2} \beta_{3} \Omega_{R}}{\mu_{R}^{2}\left(\alpha_{2}+\mu_{R}\right)}\right) \leq-\mu_{R}\left(1-R_{0 R}\right)
\end{gathered}
$$

The above inequalities (49) and (50) holds and remains negative if and only if $R_{0 H}<1$ and $R_{0 R}<1$. This proves the Lemma 1 .

We proceed in proving our theorem and we have that:

Since $R_{0 H}<1$ and $R_{0 R}<1$ holds;

Then, 


$$
\begin{aligned}
& R_{0 H} R_{0 R}<1 \\
\Rightarrow R_{G}^{2}= & R_{0 H} R_{0 R}<1 \\
\therefore & R_{G}<1
\end{aligned}
$$

Holds

This proves theorem $2 \square$

\subsubsection{LEMMA 1:}

The Disease-Free equilibrium of the Human and the Rodent's population is asymptotically stable when $R_{0 H}<1$ and $R_{0 R}<1$ respectively $\square$

\subsection{SENSITIVITY ANALYSIS OF THE RODENT-HUMAN MODEL}

The Sensitivity analysis of the Rodent-Human interactive model of Lassa fever transmission has to do with the determination of the parameters most responsible for the transmission and spread of the lassa haemorraghic disease.

It is an analysis of the reproduction number $R_{G}=\sqrt{R_{0 H} R_{0 R}}$ of the model which checks for the variation and effect of a parameter on $R_{G}$ when increased or decreased.

The values (numerical or variable) or parameter obtained after carrying out a sensitivity analysis on $R_{G}$ are called SENSITIVITY INDEX/INDICES (S.I)

\subsubsection{DEFINITION 1:}

The Normalized forward-sensitivity index of a variable $Z$ that depends differentiably on a parameter $W$ is defined as:

$$
\chi_{W}^{Z}=\frac{\partial Z}{\partial W} \cdot \frac{W}{Z}
$$

We would now compute the sensitivity indices of the basic reproduction number $R_{G}$, with respect to the model parameters. 


\subsubsection{Sensitivity Index for $\beta_{1}$}

The normalized forward sensitivity index of $\beta_{1}$ is given by:

$$
\begin{gathered}
\chi_{\beta_{1}}^{R_{G}}=\frac{\partial R_{G}}{\partial \beta_{1}} \cdot \frac{\beta_{1}}{R_{G}} \\
R_{G}=\sqrt{\frac{\alpha_{1} \alpha_{2} \beta_{1} \beta_{3} \Omega_{H} \Omega_{R}}{\mu_{H} \mu_{R}^{2}\left(\alpha_{1}+\mu_{H}\right)\left(\mu_{H}+\delta+\eta\right)\left(\alpha_{2}+\mu_{R}\right)}}
\end{gathered}
$$

By computing and evaluating the derivatives in (52) appropriately, we have;

$$
\begin{gathered}
\frac{\partial R_{G}}{\partial \beta_{1}}=\frac{1}{2 \beta_{1}} R_{G} \\
\therefore \chi_{\beta_{1}}^{R_{G}}=\frac{\partial R_{G}}{\partial \beta_{1}} \cdot \frac{\beta_{1}}{R_{G}}=\frac{1}{2 \beta_{1}} R_{G} \cdot \frac{\beta_{1}}{R_{G}} \\
\Rightarrow \chi_{\beta_{1}}^{R_{G}}=+\frac{1}{2}
\end{gathered}
$$

This gives us the sensitivity index for $\beta_{1}$.

Similarly, we implement this method all through to obtain the sensitivity indices of all other parameters in the basic reproduction number. Thus having the parameters' indices below as:

$$
\begin{aligned}
& \chi_{\beta_{3}}^{R_{G}}=+\frac{1}{2} ; \quad \chi_{\alpha_{2}}^{R_{G}}=+\frac{1}{2} \frac{\mu_{R}}{\left(\alpha_{2}+\mu_{R}\right)} ; \quad \chi_{\Lambda_{H}}^{R_{G}}=+\frac{1}{2} ; \quad \chi_{\Lambda_{R}}^{R_{G}}=+\frac{1}{2} ; \chi_{\eta}^{R_{G}}=-\frac{1}{2} \frac{\eta}{\left(\mu_{H}+\delta+\eta\right)} ; \\
& \chi_{\mu_{H}}^{R_{G}}=-\frac{1}{2}\left[1+\frac{\mu_{H}}{\left(\alpha_{1}+\mu_{H}\right)}+\frac{\mu_{H}}{\left(\mu_{H}+\delta+\eta\right)}\right] ; \quad \chi_{\mu_{R}}^{R_{G}}=-\frac{1}{2}\left[2+\frac{\mu_{R}}{\left(\alpha_{2}+\mu_{R}\right)}\right] \\
& \chi_{\delta}^{R_{G}}=-\frac{1}{2}\left[\frac{\delta}{\left(\mu_{H}+\delta+\eta\right)}\right] ; \quad \chi_{\alpha_{1}}^{R_{G}}=+\frac{\mu_{H}}{2\left(\alpha_{1}+\mu_{H}\right)} ;
\end{aligned}
$$

\subsection{Interpretation of Sensitivity Indices}

In the results obtained from the sensitivity analysis and computation, the "positive" sensitivity indices of the model parameters in $R_{G}$ indicates an "increase (or decrease)" in the value of these parameters being computed will bring about an "increase (or decrease)" in $R_{G}$; While on the other hand, the "negative" sensitivity indices interprets that an "increase (or decrease)" in the value of this parameter will bring about a/an decrease (or increase) in $R_{G}$. 
For example, $\chi_{\beta_{1}}^{R_{G}}=+\frac{1}{2}$ suggests that increasing (or decreasing) the effective contact rate between the human and the rodents by $x \%$ (or say 30\%) will bring about an increase (or decrease) of $\frac{x}{2} \%$ (or say $15 \%$ ) in $R_{G}$.

For the Negative sign, $\chi_{\mu_{R}}^{R_{G}}=-\frac{1}{2}\left[2+\frac{\mu_{R}}{\left(\alpha_{2}+\mu_{R}\right)}\right]$ suggests that increasing (or decreasing) the hunting/natural death rate of the rodents by $x \%$ (or say $30 \%$ ) will bring about a decrease (or increase) of $x\left[1+\frac{\mu_{R}}{\left(\alpha_{2}+\mu_{R}\right)}\right] \%\left(\right.$ or say $\left.30\left[1+\frac{\mu_{R}}{\left(\alpha_{2}+\mu_{R}\right)}\right] \%\right)$ or $9 \%$ numerically in $R_{G}$.

As a result of these, carrying out a sensitivity analysis of the proposed model projects a very good view into the transmission dynamics of the disease.

Most especially, it aids the public health authorities in concentrating on an appropriate intervention strategy for preventing and controlling the spread of the disease.

We now have the sensitivity indices table as:

TABLE 3.5.1

\begin{tabular}{ll}
\hline PARAMETER & SENSITIVITY \\
& INDICES (S.I) \\
\hline$\Omega_{H}$ & + \\
$\Omega_{R}$ & + \\
$\beta_{1}$ & + \\
$\beta_{2}$ & + \\
$\alpha_{1}$ & + \\
$\alpha_{2}$ & - \\
$\eta$ & - \\
$\mu_{H}$ & - \\
$\mu_{R}$ & - \\
$\delta$ & \\
\hline
\end{tabular}




\subsection{NUMERICAL RESULTS AND SIMULATIONS}

In this section, we have studied, examined and analyzed the analytical/simulated results of the rodent-human interactive model of lassa haemorraghic fever. This is to verify the sensitivity analysis carried out on the model, to establish our theoretical findings, also to study, analyze, and interpret the dynamical behavior of the system.

Using the Runge-kutta-felhberg of fourth-fifth order (RKR-45) with degree four interpolant in the Maple software, we have simulated the entire and single compartments of the model with respect to the values of the state variables (initial conditions) and by varying some cogent parameters in the model. The values, dimensions, and sources of these parameters have been presented in the Table 4.0.1 below.

Most of these parameters were clinical data which have been extracted from prominent literature while some were reasonably estimated and assumed.

TABLE 4.0.1

\begin{tabular}{lll}
\hline $\begin{array}{l}\text { STATE } \\
\text { VARIABLES }\end{array}$ & VALUE & SOURCE \\
\hline$S_{H}(t)$ & 5,500 & Assumed \\
$E_{H}(t)$ & 1,650 & Estimated \\
$\boldsymbol{I}_{H}(t)$ & 650 & Assumed \\
$R_{H}(t)$ & 100 & Assumed \\
$S_{R}(t)$ & 60 & Estimated \\
$E_{R}(t)$ & 30 & Estimated \\
$\boldsymbol{I}_{R}(t)$ & 10 & Assumed \\
\hline
\end{tabular}

\begin{tabular}{llll}
\hline PARAMETER & VALUE & DIMENSION & SOURCE \\
\hline$\Omega_{H}$ & 1.2 & Humans $\times$ day & Estimated \\
$\Omega_{R}$ & 0.70 & Rodents $\times$ day $^{-1}$ & Estimated \\
$\beta_{1}$ & 0.04 & Dimensionless & Assumed \\
$\beta_{2}$ & 0.04 & Dimensionless & Assumed \\
\hline
\end{tabular}




\begin{tabular}{llll}
\hline$\beta_{3}$ & 0.005 & Dimensionless & Assumed \\
$\alpha_{1}$ & 0.10 & Day $^{-1}$ & NCDC (2019) [26] \\
$\alpha_{2}$ & 0.057 & Day $^{-1}$ & NCDC (2019) [26] \\
$\phi$ & 0.83 & Dimensionless & Assumed \\
$\Psi_{1}$ & 0.091193 & Dimensionless & Estimated \\
$\Psi_{2}$ & 0.32 & Dimensionless & Estimated \\
$\eta$ & 0.030 & Day $^{-1}$ & NCDC(2019) [26] \\
$\mu_{H}$ & 0.0003465 & Day $^{-1}$ & WHO (2018) [13] \\
$\mu_{R}$ & 0.00261026 & Day $^{-1}$ & WHO (2020) [11] \\
$\delta$ & 0.01710615 & Day $^{-1}$ & NCDC (2019) [26] \\
$\rho$ & 0.0085 & Day $^{-1}$ & Bakare E.A et al. (2020) [32] \\
\hline
\end{tabular}

The Figure 1-8 shows the combined compartmental plots of the human and the rodent population respectively with respect to the variations of parameters in the table 4.0.1.

Figures 9-15 graphically illustrates the behavior of the single compartments (susceptible, exposed, infected and recovered) when some parameters (most especially the saturation factor) is increased or decreased. These illustrations were presented in the Figures 1 to 15 below:

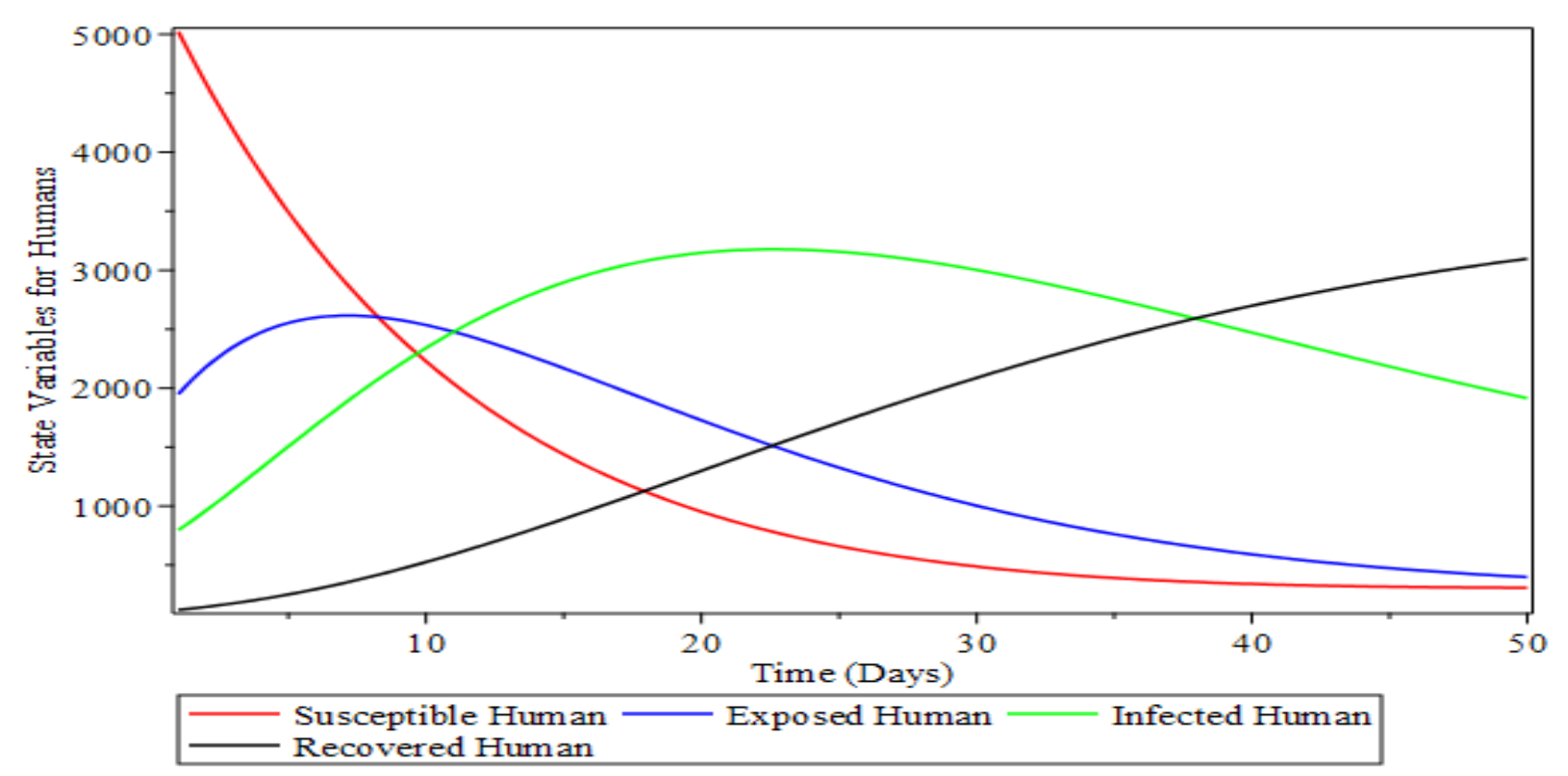




\section{Fig 1}

The Dynamical behavior of the human state variables with $\Omega_{H}=1.2, \Psi_{1}=0.091193, \mu_{H}=0.0003465, \rho=0.0085, \alpha_{1}=0.10, \delta=0.01710615, \eta=0.030$ showing that all the compartments co-exists, thereby causing the system to approach an Endemic state.

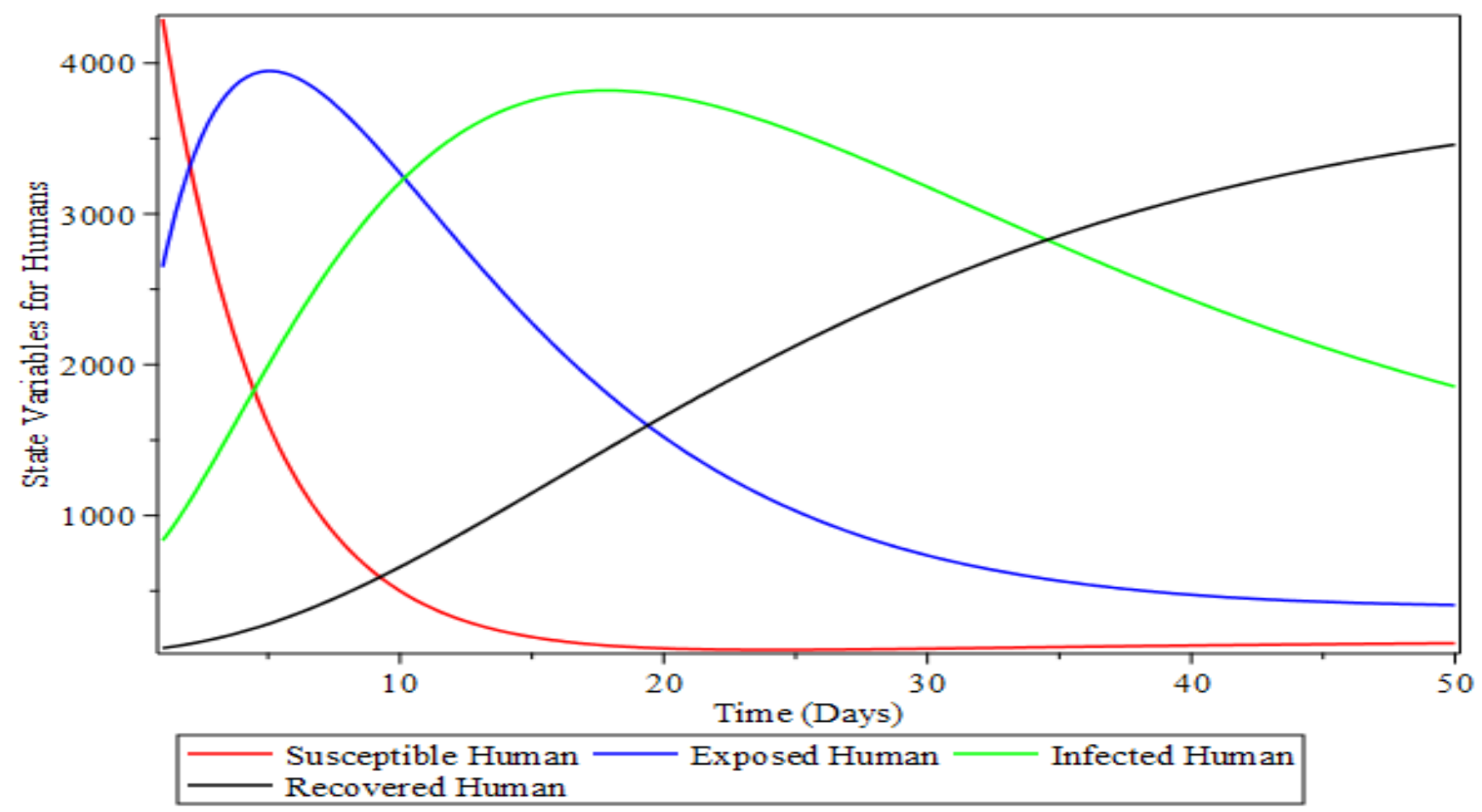

Fig 2

The Dynamical behavior of the humans' state variables with $\Omega_{H}=10, \Psi_{1}=0.25, \mu_{H}=0.0003465, \rho=0.0085, \alpha_{1}=0.10, \delta=0.01710615, \eta=0.030 ;$ showing that all compartments co-exists, thereby causing the system to approach an Endemic state. 


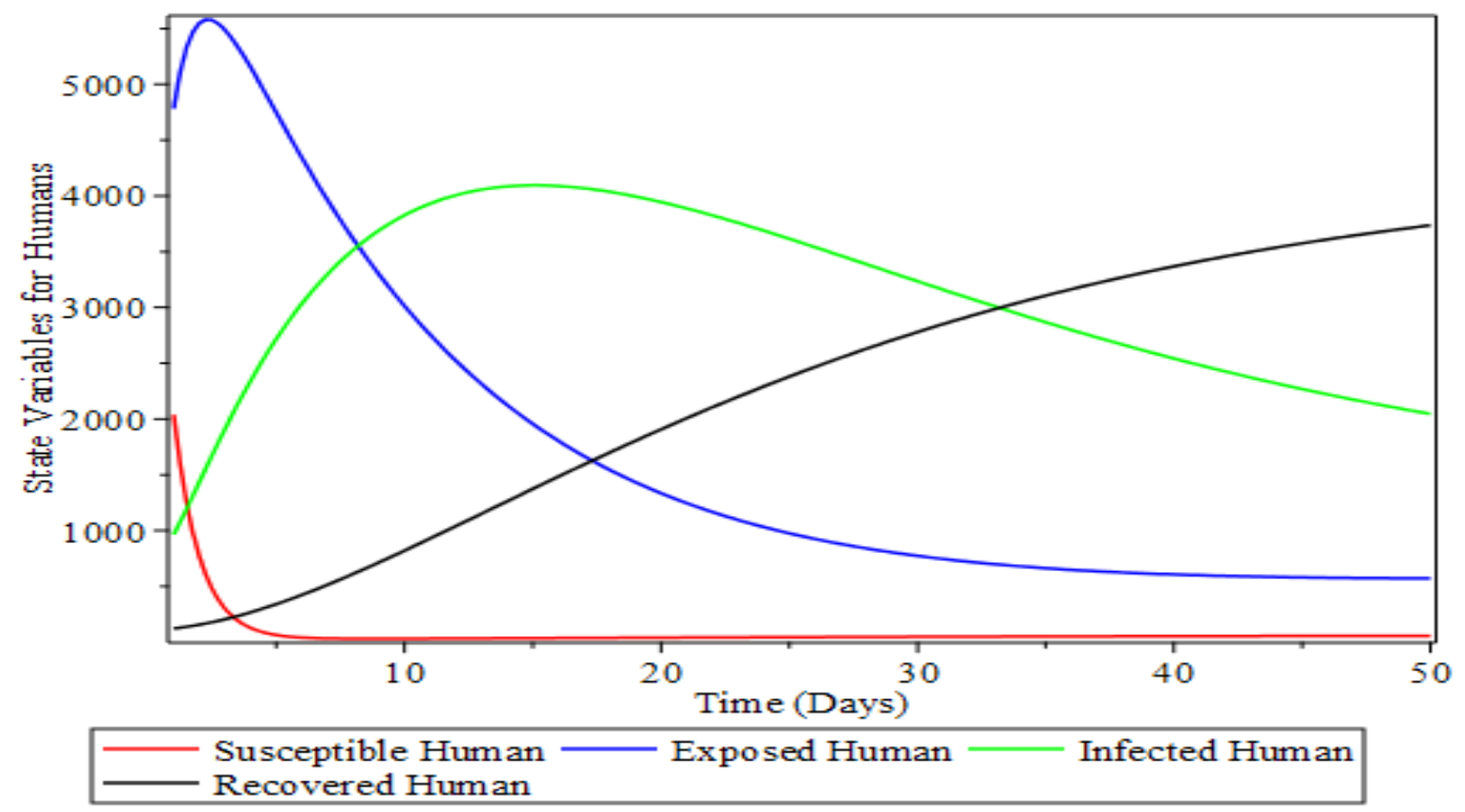

Fig 3

The Dynamical behavior of the humans' state variables with $\Omega_{H}=25, \Psi_{1}=1, \mu_{H}=0.0003465, \rho=0.0085, \alpha_{1}=0.10, \delta=0.01710615, \eta=0.030 ; \quad$ showing that all compartments co-exists, thereby causing the system to approach an Endemic state.

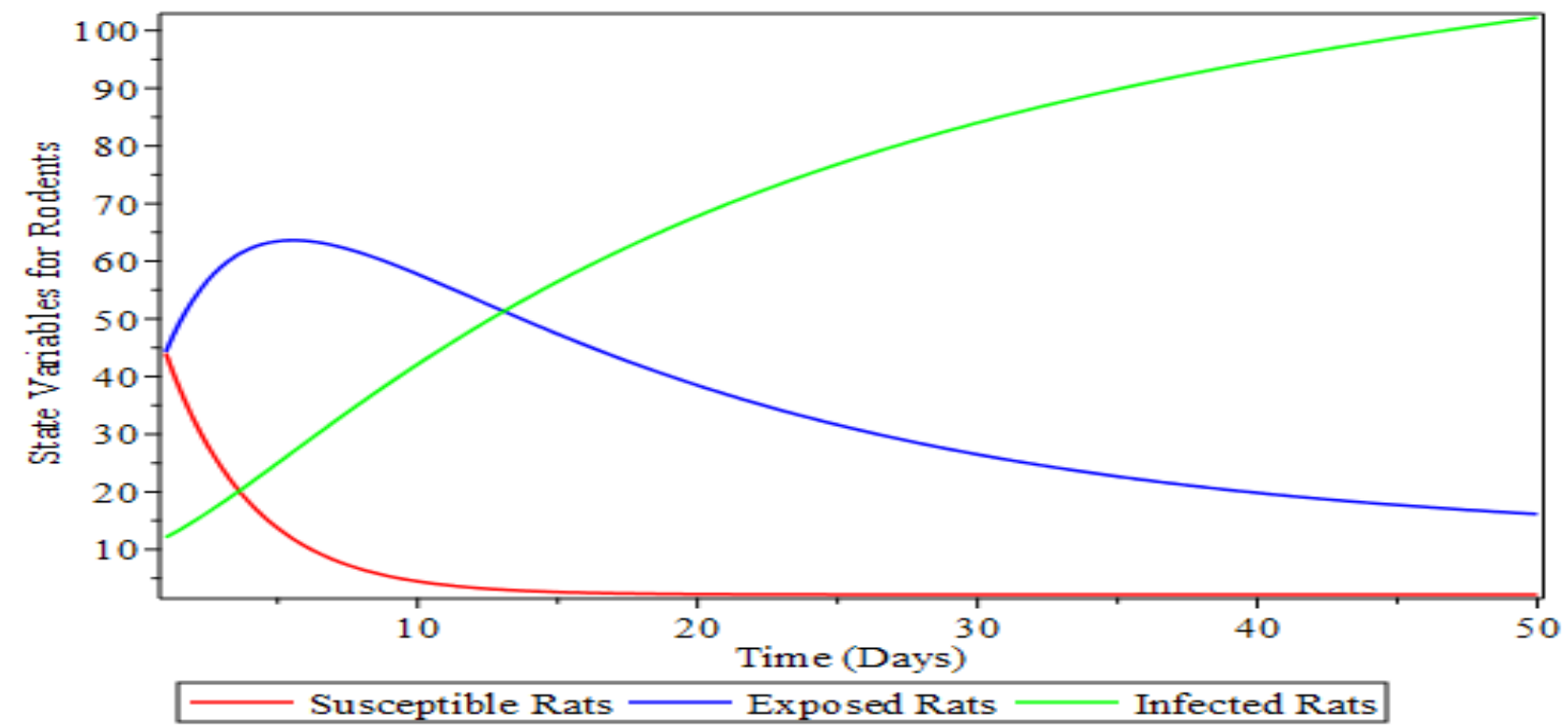

Fig 4

The Dynamic behavior of the Rodents' state variables with $\Omega_{R}=0.70, \Psi_{2}=0.32, \mu_{R}=0.00261026, \alpha_{2}=0.057$ showing that all the compartments coexists 
as well, causing the system to approach an Endemic state. Also the susceptible population tends to wipe out as a particular time.

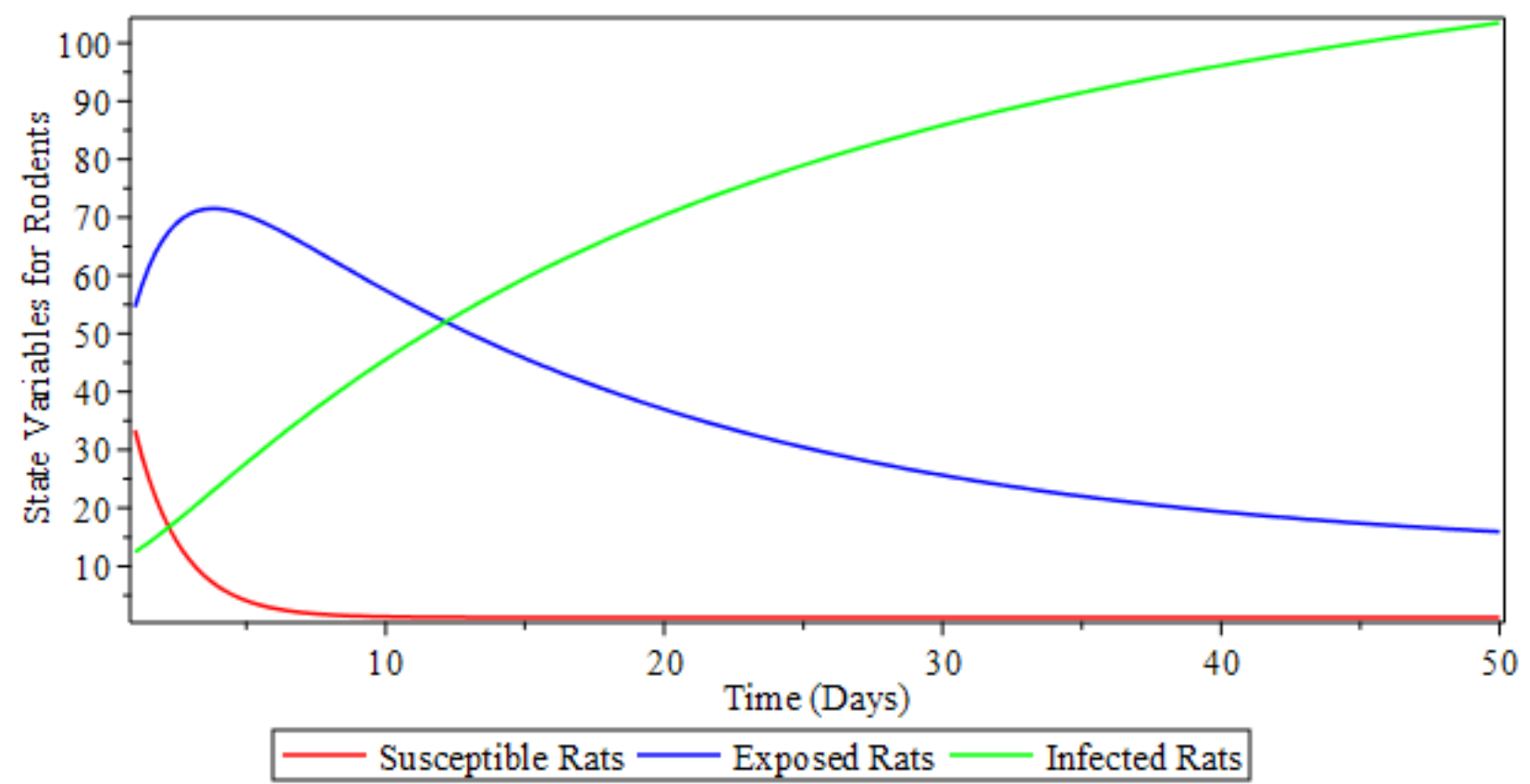

\section{Fig 5}

The Dynamic behavior of the Rodents' state variables with respect to $\Omega_{R}=0.70, \Psi_{2}=0.6, \mu_{R}=0.00261026, \alpha_{2}=0.057$, when the force of infection is doubled. This shows that with this increase in the force of infection, the susceptibility rate drops sharply while the infected rats' population shoots up or increases up to a steady state swiftly.

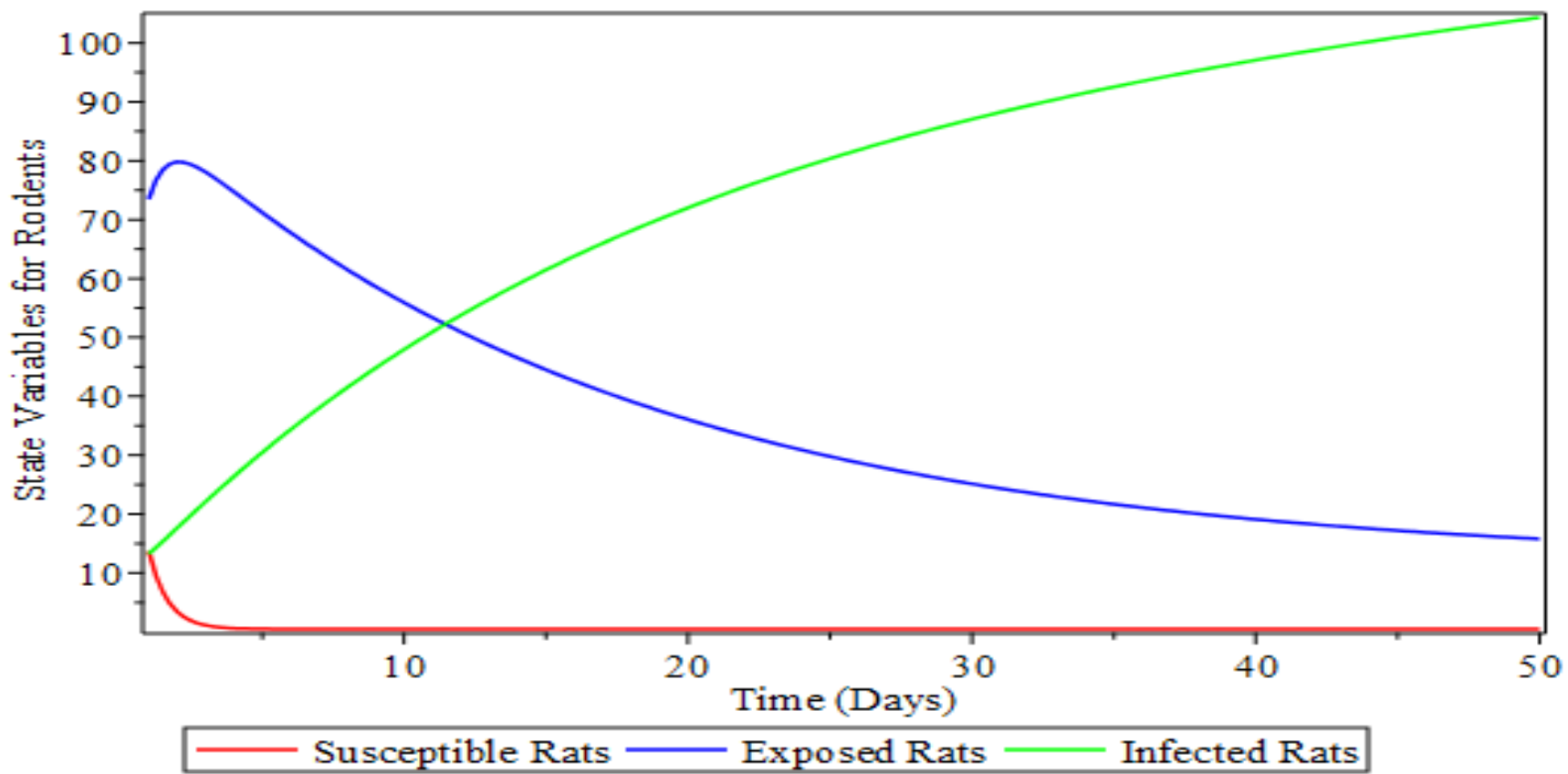




\section{Fig 6}

The Dynamic behavior of the Rodents' state variables with $\Omega_{R}=0.70, \Psi_{2}=1.5, \mu_{R}=0.00261026, \alpha_{2}=0.057$, tripling the force of infection. This shws that with this increase in the force of infection, the susceptibility rate drops sharply while the infected rats' population shoots up or increases up to a steady state.

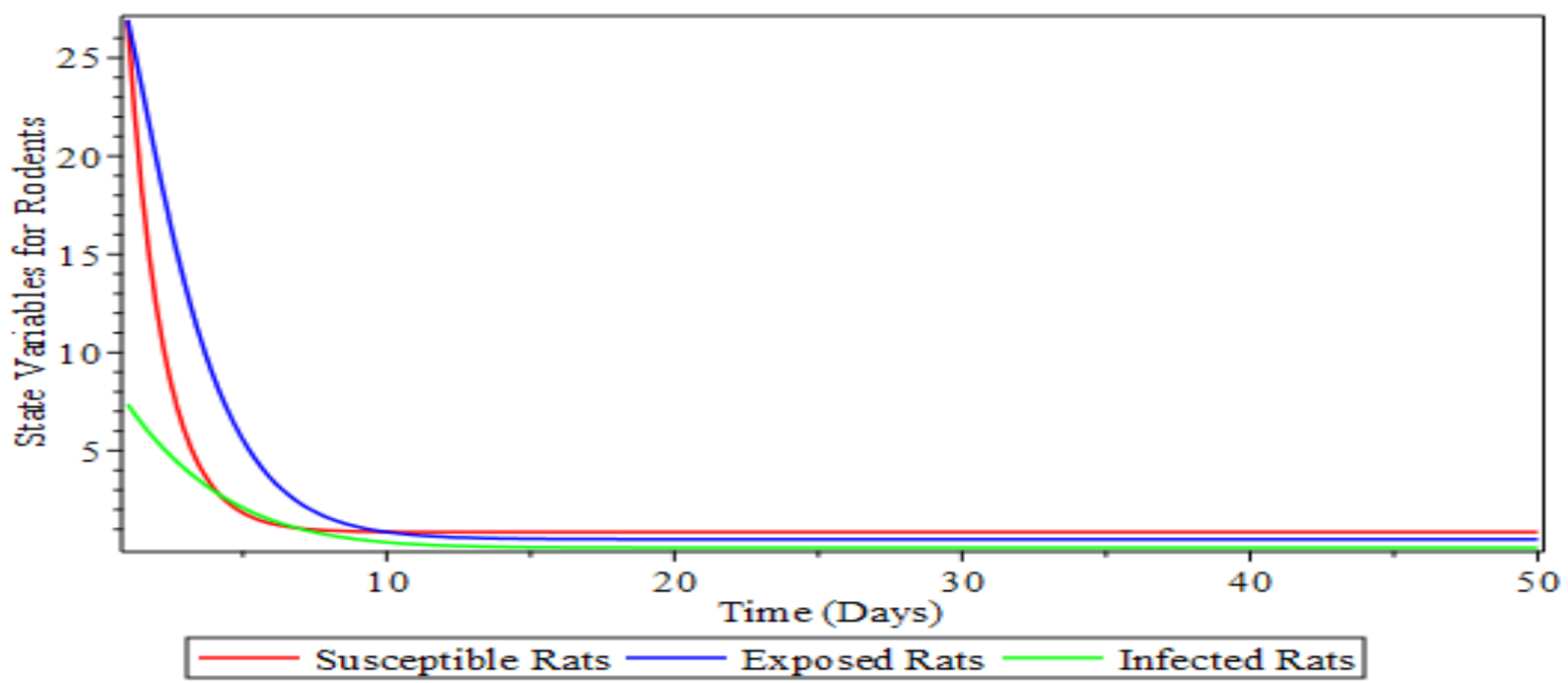

Fig 7

The Dynamic behavior of the Rodents' state variables with $\Omega_{R}=0.70, \Psi_{2}=1.5, \mu_{R}=0.5, \alpha_{2}=0.057$ showing that with an increase in the death rate by natural cause, hunting, or other human control measures, the entire population most especially the infected population decline sharply and drastically, causing the system to approach a Disease-free equilibrium (DFE).

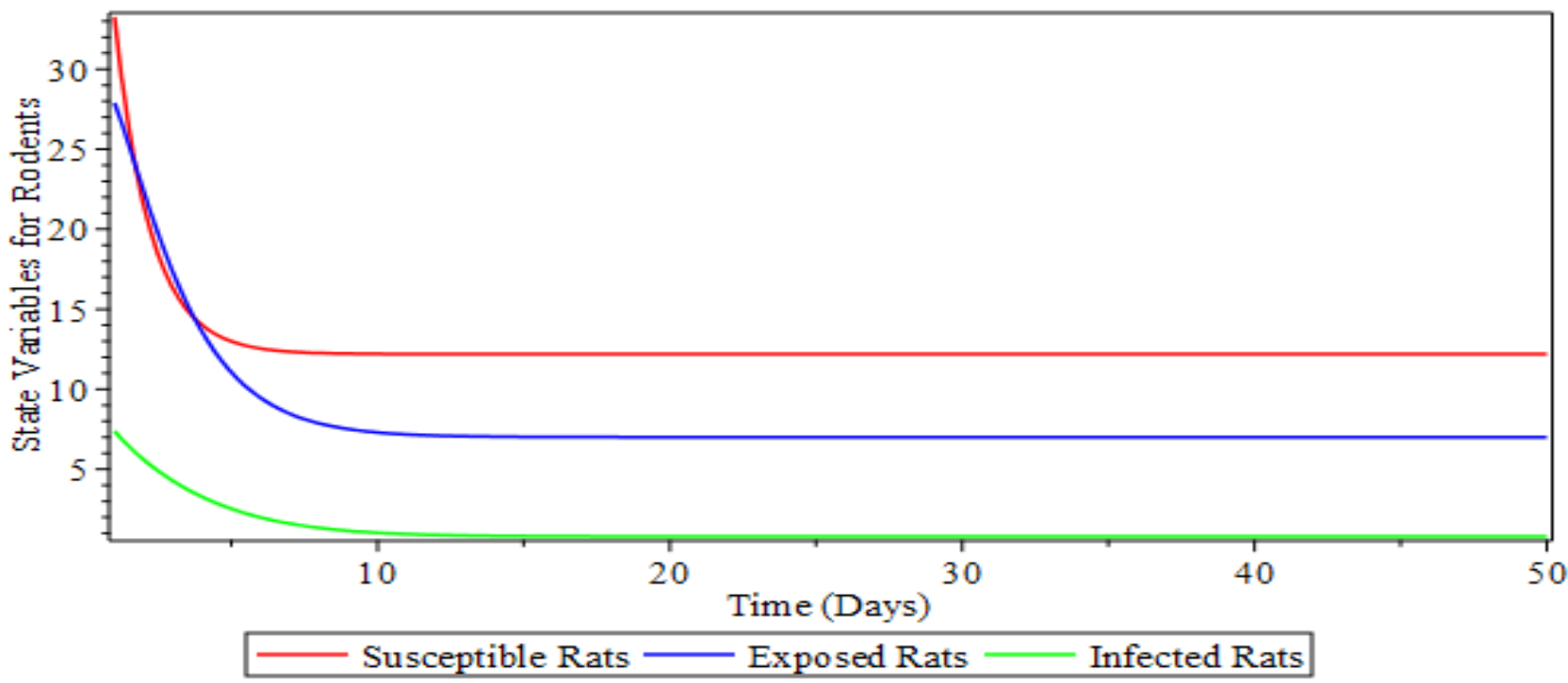


Fig 8

The Dynamical Behavior of the Rodents' state variables with $\Omega_{R}=10, \Psi_{2}=1.5, \mu_{R}=0.5, \alpha_{2}=0.057$ showing that irrespective of an increase in the recruitment rate of the rodents, there is still a sharp and drastic decrease due to the increased death rate

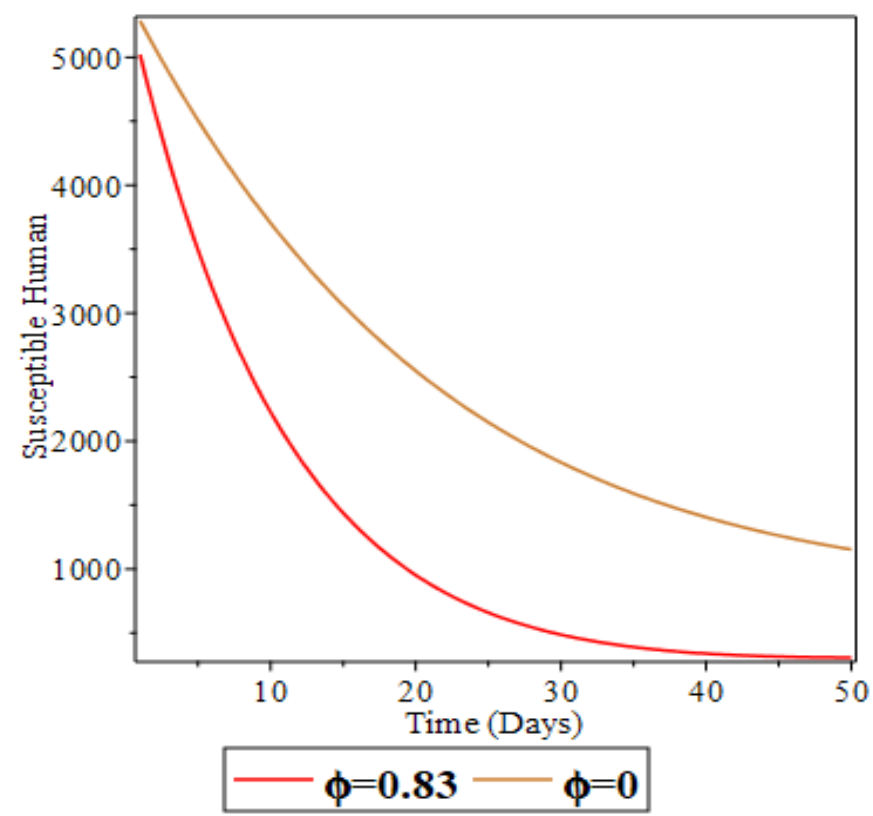

Fig 9

The Changing effect of the susceptible human with and without the "saturation factor" $\phi$.This shows that the rate of human susceptibility to Lassa fever is reduced when saturation is being introduced

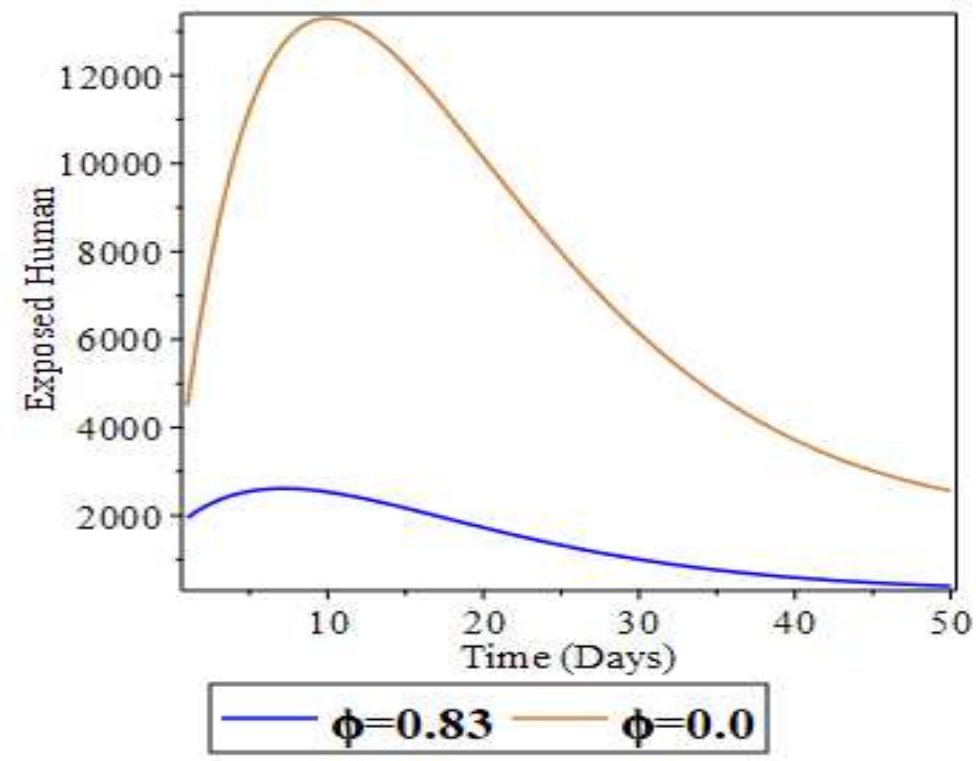




\section{Fig 10}

The changing effect of the exposed human with and without the "saturation factor" $\phi$.This shows that the rate of human exposure to the lassa virus from the "Mastomys" is reduced when saturation sets in.

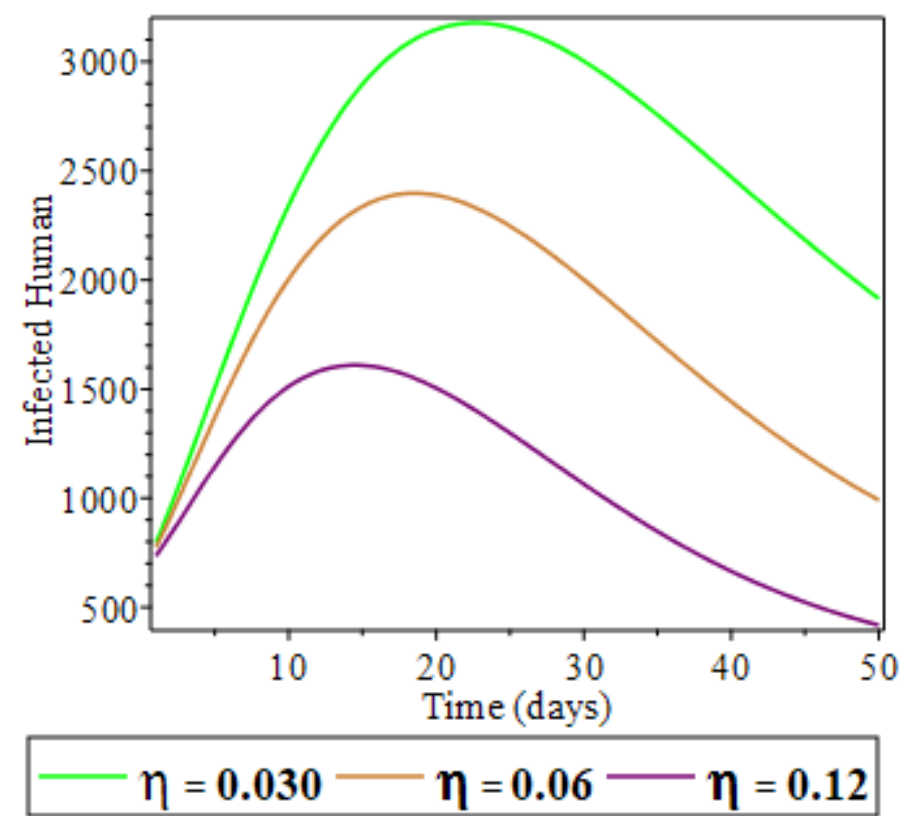

\section{Fig 11}

The changing effect of the infected human with increase rate of treatment via the administration of "Ribavirin" which shows that the early administration and implementation of antiviral therapy can drastically drop the population in the infected compartment

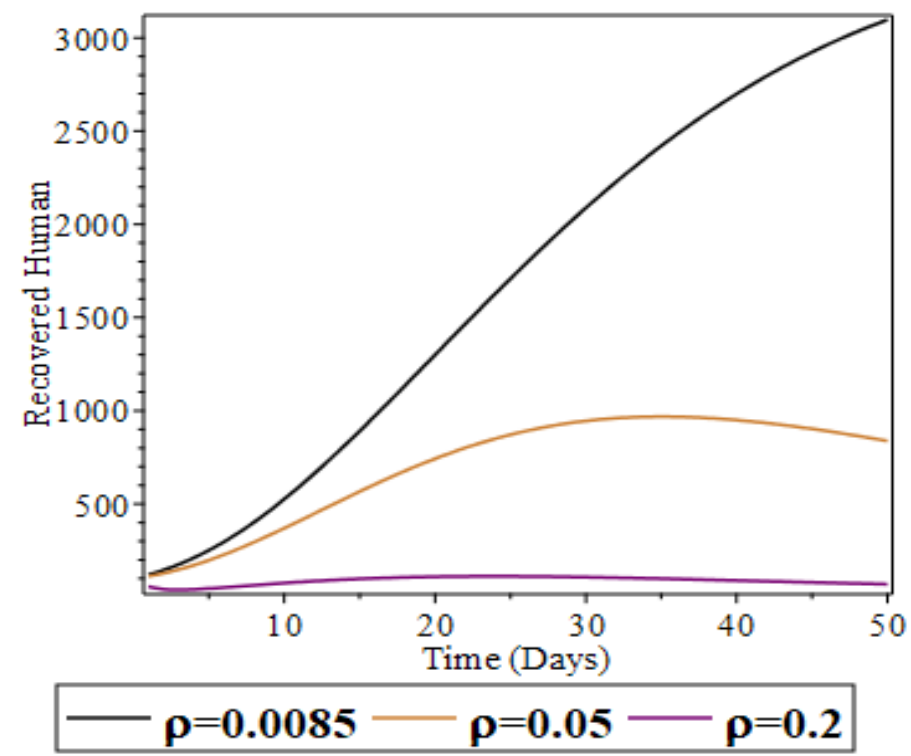




\section{Fig 12}

The changing effects on the Recovered human population by varying loss of immunity

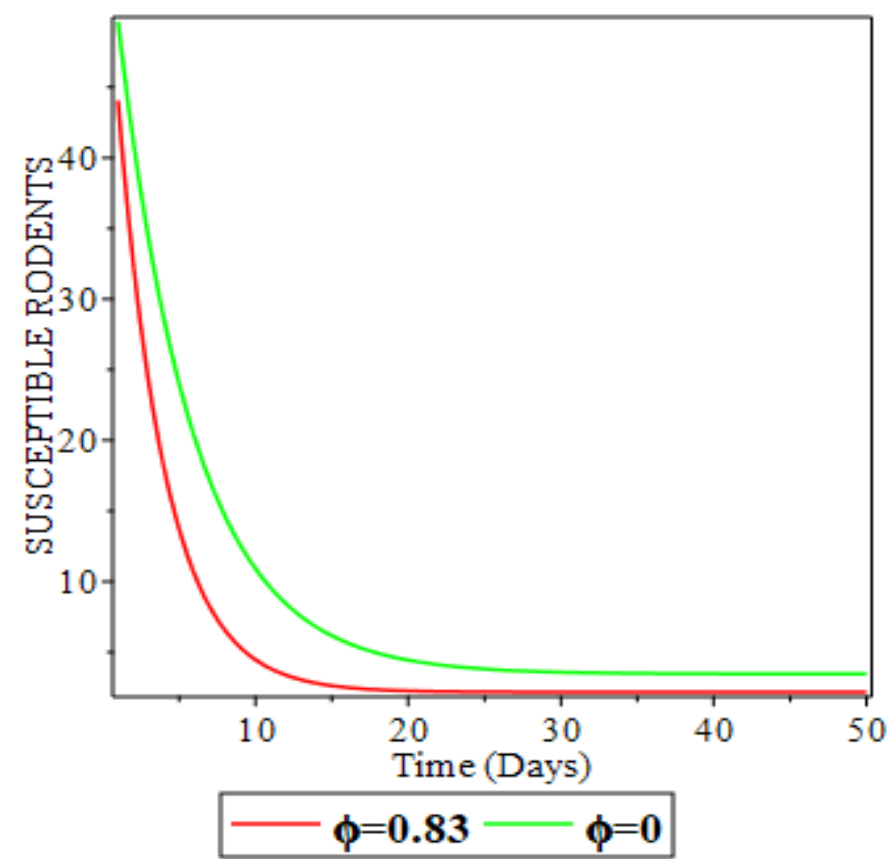

\section{Fig 13}

The Changing effect of the susceptible rodents with and without the "saturation factor" $\phi$.This shows that the rate of rodents' susceptibility to the wild rodents is reduced when saturation is being introduced

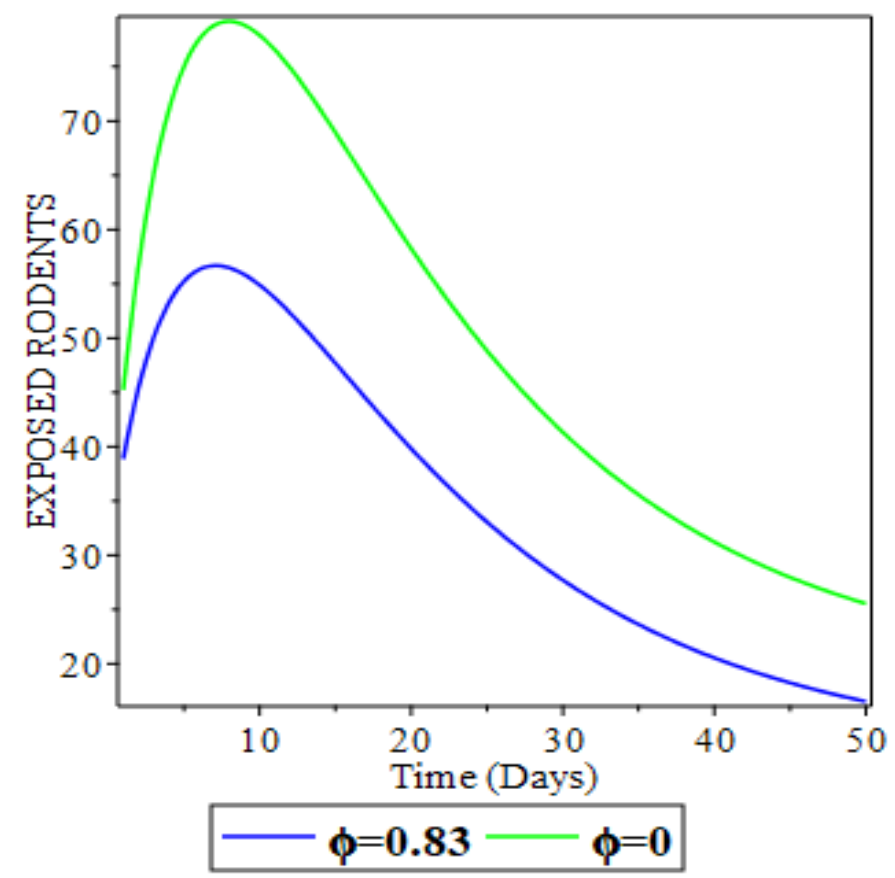




\section{Fig 14}

The changing effect of the exposed rodents with and without "saturation factor" $\phi$ which shows that the rate of rodents' exposure to wild rodents and the population of the exposed rodents is reduced in the presence of the "saturation factor"

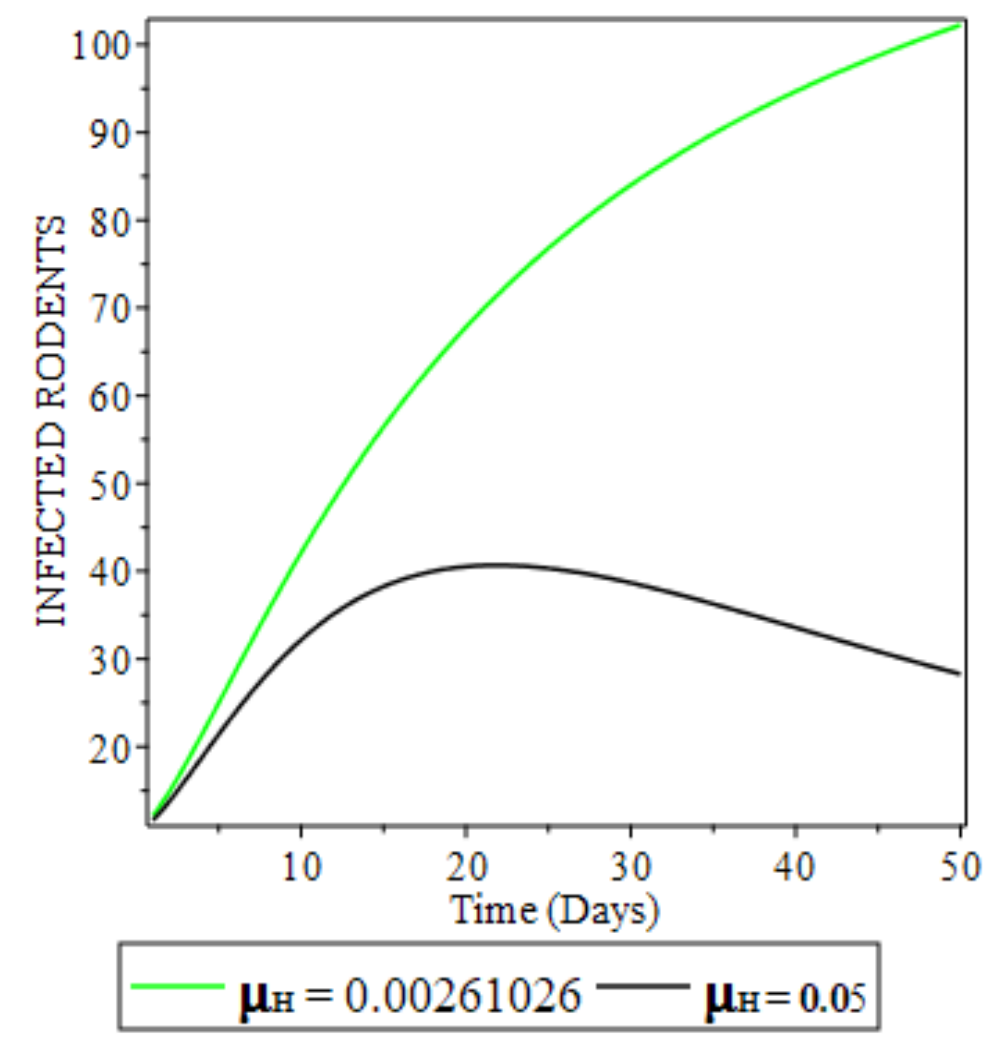

\section{Fig 15}

The changing effect of the infected when death rate (by hunting or other control measures, and natural cause) is increased.

\subsection{DISCUSSION AND INTERPRETATION OF SIMULATED RESULTS}

\subsubsection{Combined Compartments}

We have simulated the model using the initial conditions (State variables) on the table 4.0.1 with the parameters as well. To check the dynamical behavior and changes in the model, we now have to vary some of these parameters on the table above.

The Figure 1 illustrates the combined graphs of all the human compartments (susceptible human, exposed human, infected human, and recovered human), using the standard initial conditions and 
parameter values on the table 4.0.1. From the dynamical behavior of the system in Figure 1, it is observed that the infected and the exposed humans population is increased at the initial phase (Instant-time) but on the long run the exposed decreases totally to a steady state (logistic decay) and the infected falls to a steady state as well.

Meanwhile, the entire population tends to be susceptible at the initial phase showing the wellposedness of our model, and the human population starts to recover in less than 35 days (The system hence approaches an Endemic state).

Figure 2 illustrates the dynamical behavior of all the human compartments when the human recruitment rate $\Lambda_{H}$ and the force of infection $\Psi_{1}$ are varied. By varying these parameters, we observed that the humans get more exposed and infected; the susceptible human population drops sharply and drastically in less than 20 days but the humans tends to recover in less than 30 days. All these show that all the compartments co-exist in the system causing the system to approach an Endemic state.

Figure 3 illustrates the behavior of the system when the force of infection $\Psi_{1}$ is unity $\left(\Psi_{1}=1\right)$ and the recruitment rate is increased. Here the human population gets exposed really fast at instant time (again, showing the well-posedness of the model), the infected human population also increases and later stabilizes after a month and some days, causing the susceptible population to drop drastically within 10 days.

Similarly, in Figure 4, 5, and 6, the dynamic behavior of the rodent system with respect to the initial conditions and parameters values on Table 4.0.1, doubling and tripling the force of infection for the figures 5 and 6 respectively; shows that the rodents tends to be exposed at instant time, causing them to get infected in less than 10, 5, and 2 days respectively; but the infected population on the long run reaches a steady state. Also, the susceptible population tends to wipe out in 15 days leaving only the exposed and the infected population in the system (Endemic equilibrium).

In Figure 7 and 8, it was observed that the increment of the death rate by hunting, human control measures and natural cause tends to move the rodents' system to a disease-free equilibrium swiftly irrespective of the increase in their recruitment rate in Fig 8. 


\subsubsection{Single Compartment graphs}

Figures 9, 10, 13, 14 have been used to validate and verify the effect of the saturation parameter/factor in this proposed model. The changing effect or dynamical behavior of the susceptible and exposed human shows that with the presence of "saturation" (This could be some control measures like the hunting of wild rodents in the case of the rodent population (Figures 13 and 14), antibody response to the virus, or the early administration of antiviral like "Ribavirin"), the populations of the susceptible and exposed human is reduced drastically; causing the system to approach a DFE.

Similarly to the above, the dynamical behavior was tested for the variations in recovery rate as a result of antiviral administration in Figure 11. This shows that increase in recovery rate drastically reduces the population of the infected human. While loss of immunity in Figure 12 brings about the re-susceptibility of the recovered humans

Lastly, the Figure 15 shows the changing effect of the infected rodent population when the death rate by hunting/human control or natural cause is induced. This vividly shows that hunting and killing wild rodents would cause the entire system to approach a DFE; as the infected wild rodents would be drastically reduced.

\subsection{CONCLUSION}

In this research, we have studied an interactive rodent-host model unprecedented, built on a 7dimensional nonlinear system of ODE, to describe and capture the transmission dynamics and dynamic behavior of the Lassa Haemorrhagic fever. Qualitative analyses have been conveyed on this model viz: stability analysis and sensitivity analysis. Lastly, we have simulated the model using clinical, estimated, and assumed data extracted from WHO, Nigerian centre for disease control (NCDC), and other prominent literatures.

We conclude from our results and analysis that the presence of "saturation" (this could stem from treatment or control measures hindering the epidemic of the virus in a susceptible population) instantly causes the system to approach a LHF-free equilibrium. That is, the implementation of an appropriate control plan (quarantining, mass testing/diagnosis and 
treatment, mass education, and sensitization of the masses) would have significant effect on the eradication of the disease.

Also from the stability and sensitivity analysis, the most sensitive parameters which require the attention of the control body (Healthcare) are the rodents' recruitment rate $\Omega_{R}$ and the effective contact rates $\beta_{1}, \beta_{2}$, and $\beta_{3}$ which leads to the lassa haemorrhagic virus infection. As this would cause the basic reproduction number remain less than unity when controlled.

We also recommend for the public healthcare that early intervention and timely treatment with "Ribavirin" through raising the efficiency and enlarging the capacity of treatment would eradicate the disease instantaneously; humans should try as much possible to maintain maximum hygiene and clean environment so as not to create an avenue for these rodents in the house and also to prevent them from coming in contact with Food and grains (this could be achieved through public education schemes and sensitization on the Lassa virus); Rodent extermination and evacuation should be periodically done in households to curb the recruitment rate of Mastomys.

\subsection{DECLARATION OF INTEREST}

Authors have declared that there is no conflict of interest relevant to this research work.

\section{REFERENCES}

[1] Safronetz, D., Lopez, J. E., Sogoba, N., Traore', S. F., Raffel, S. J., Fischer, E. R., Ebihara, H., Branco, L., Garry, R. F., Schwan, T. G., \& Feldmann, H. (2010) Detection of Lassa virus, Mali. Emerging infectious $\quad$ diseases, 16(7), 1123-1126. https://doi.org/10.3201/eid1607.100146

[2] Akinade, Mary, Afolabi, Ayodeji \& Kimathi, Mark. (2019) Mathematical Modeling and Stability Analyses of Lassa fever Disease with the Introduction of the Carrier Compartment. 9. 45-62. 10.7176/MTM.

[3] Centre for Disease Control: Lassa fever Reports. Available at: https://www.cdc.gov/vhf/lassa/index.html

[4] Onuorah, M.O., Akinwande, N.I., Nasir M.O., \& Ojo M.S (2016) Sensitivity analysis of Lassa fever model, International Journal of Mathematics and statistics studies; 4(1): pp. 30-49 
[5] Joseph B. McCormick, Isabel J. King, Patricia A. Webb, Karl M. Johnson, Renie O'Sullivan, Ethleen S. Smith, Sally Trippel, Tony C. Tong, A Case-Control Study of the Clinical Diagnosis and Course of Lassa Fever, The Journal of Infectious Diseases, Volume 155, Issue 3, March 1987, Pages 445-455, https://doi.org/10.1093/infdis/155.3.445

[6] ProMed International Society for Infectious Diseases: Lassa fever - West Africa (43): Liberia (2019). Available at: https://promedmail.org/promed-post/?id=20191207.6828798

[7] ProMed International Society for Infectious Diseases: Lassa fever - Nigeria, Liberia (2014): Available at: https://promedmail.org/promed-post/?id=20140328.2363217

[8] ProMed International Society for Infectious Diseases: Lassa fever - West Africa (04), Liberia (2017): Available at: https://promedmail.org/promed-post/?id=20170209.4827934

[9] World Health Organization - Disease Outbreak news and Reports (Lassa fever), 20 February 2020. Available at: https://www.who.int/csr/don/20-february-2020-lassa-fevernigeria/en/

[10] Centre for Disease control (CDC) - Lassa fever Transmission. Available at: https://www.cdc.gov/vhf/lassa/transmission/index.html

[11] World Health Organization - Disease Outbreak news and Reports (Lassa fever), 28 June 2020. Available at: https://www.who.int/csr/don/28-june-2017-lassa-fever-nigeria/en/

[12] Amy Maxmen (2018); News in focus - Deadly outbreak tests Nigerian health agency, Nature, Vol. 555, pp. 421-422 Available at: https://media.nature.com/original/magazineassets/d41586-018-03171-y/d41586-018-03171-y.pdf

[13] World Health Organization - Disease Outbreak news and Reports (Lassa fever, Nigeria), 20 April 2018. Available at: https://www.who.int/csr/don/20-april-2018-lassa-fever-nigeria/en/

[14] Ayodamola Owoseye (2018) Nigeria's death toll rises to 110; Premium times Newspaper; $\quad$ March $62018 . \quad$ Available at: https://www.premiumtimesng.com/news/headlines/260922-lassa-fever-nigerias-death-toll-rises$\underline{110 . h t m l}$ 
[15] Vanguard News Paper; Lassa fever deaths rise to 110 in Nigeria - official, March 6, 2018. Available at: https://www.vanguardngr.com/2018/03/lassa-fever-deaths-rise-110-nigeriaofficial/

[16] Esteva, Lourdes \& Matias, Mariano. (2001). A Model for Vector Transmitted Diseases with Saturation Incidence. Journal of Biological Systems, 09; pp. 235-245. http://doi.org/10.1142/S0218339001000414

[17] Michael A. Mikucki, Sensitivity Analysis of the basic reproduction number and other quantities for infectious disease models [Master thesis]. Colorado, North Amearica: Colorado State University, Department of Mathematics, 2012.

[18] Olaniyi Samson, Lawal Maruf \& Obabiyi Olawale (2016), Stability and Sensitivity Analysis of a Deterministic Epidemiological model with Pseudo-recover. International Journal of Applied Mathematics, 46: pp. 160-167.

[19] John Olajide Akanni \& Akinpelu Folake (2018) Sensitivity Analysis of the dynamical spread of Ebola virus disease, International journal of chemistry, mathematics and physics, 1(1): pp. $1-10$

[20] Akinpelu FO \& Akinwande R (2018) Mathematical model for Lassa fever and sensitivity analysis, Journal of scientific and engineering research, 5(6): pp. 1-9

[21] Akinade, Mary O. \& Afolabi, Ayodeji S. (2020) Sensitivity and stability analyses of a Lassa fever disease model with control strategies, IOSR Journal of Mathematics (IOSR-JM), 16(1): pp. 29-42

[22] Khan, M.A., Saddiq, S.F., Islam, S, Ilyas Khan, Sharidan Shafie (2016), Dynamic Behavior of Leptospirosis Disease with Saturated Incidence Rate. Int. J. Appl. Comput. Math 2, pp. 435-452. https://doi.org/10.1007/s40819-015-0102-2

[23] Jinhong Zhang, Jianwen Jia, \& Xinyu Song, Analysis of an SEIR Epidemic model with saturated incidence and saturated treatment function, The Scientific World Journal, volume 2014, article ID 910421, (2014), DOI: http://dx.doi.org/10.1155/2014/910421

[24] Hassan Larabi, El Houssine Labriji, Mostafa Rachik, Abdelilah Kaddar, Optimal control of an epidemic model with saturated incidence rate, Nonlinear Analysis: Modeling and Control, 17(4): pp 448-459, (2012) 
[25] Juan Liu, Bifurcation analysis for a delayed SEIR epidemic model with saturated incidence and saturated treatment function, Journal of Biological Dynamics, 13(1): pp 461-480, (2019), DOI: http://doi.org/10.1080/17513758.2019.1631965

[26] An Update of Lassa Fever outbreak in Nigeria, Nigeria Centre for Disease control (NCDC). Available at https://ncdc.gov.ng/diseases/sitreps/?cat=5\&name=An\%20update\%20of\%20Lassa\%20fever\%20 outbreak\%20in\%20Nigeria

[27] Loyinmi, A.C. \& Akinfe, T.K.:Exact solution to the family of Fisher's reaction diffusion equation using Elzaki Homotopy transformation perturbation method. Engineering Reports. 2019; e12084. DOI: https://doi.org/10.1002/eng2.12084

[28] Loyinmi, A.C., Akinfe, T.K. An algorithm for solving the Burgers-Huxley equation using Elzaki transform, SN Applied sciences, 2(7), (2020)

DOI: https://doi.org/10.1007/s42452-019-1653-3

[29] Kingsley Akinfe, Adedapo Loyinmi. Stability Analysis and Semi-Analytic Solution to a SEIR-SEI Malaria Transmission Model using He's Variational Iteration Method. Authorea. April 20, 2020 DOI: https://doi.org/10.22541/au.158739578.86609763

[30] Driessche PV and Watmough J, Reproduction numbers and sub-threshold endemic equilibria for Compartmental models of disease transmission, Mathematical Biosciences 180 (2002) pp. 2948.

[31] Diekmann, O., Heesterbeek, J.A.P. \& Metz, J.A.J. On the definition and the computation of the basic reproduction ratio $R_{0}$ in models for infectious diseases in heterogeneous populations. J. Math. Biol. 28, 365-382 (1990).DOI: https://doi.org/10.1007/BF00178324 [32] E. A. Bakare, E.B. Are, O.E. Abolarin, S. A. Osanyinlusi, Benitho Ngwu, \& Obiaderi N. Ubaka, Mathematical modeling and Analysis of Transmission dynamics of Lassa fever, Journal of Applied Mathematics, Volume 2020, Article ID 6131708, DOI:

https://doi.org/10.1155/2020/6131708 
Figures

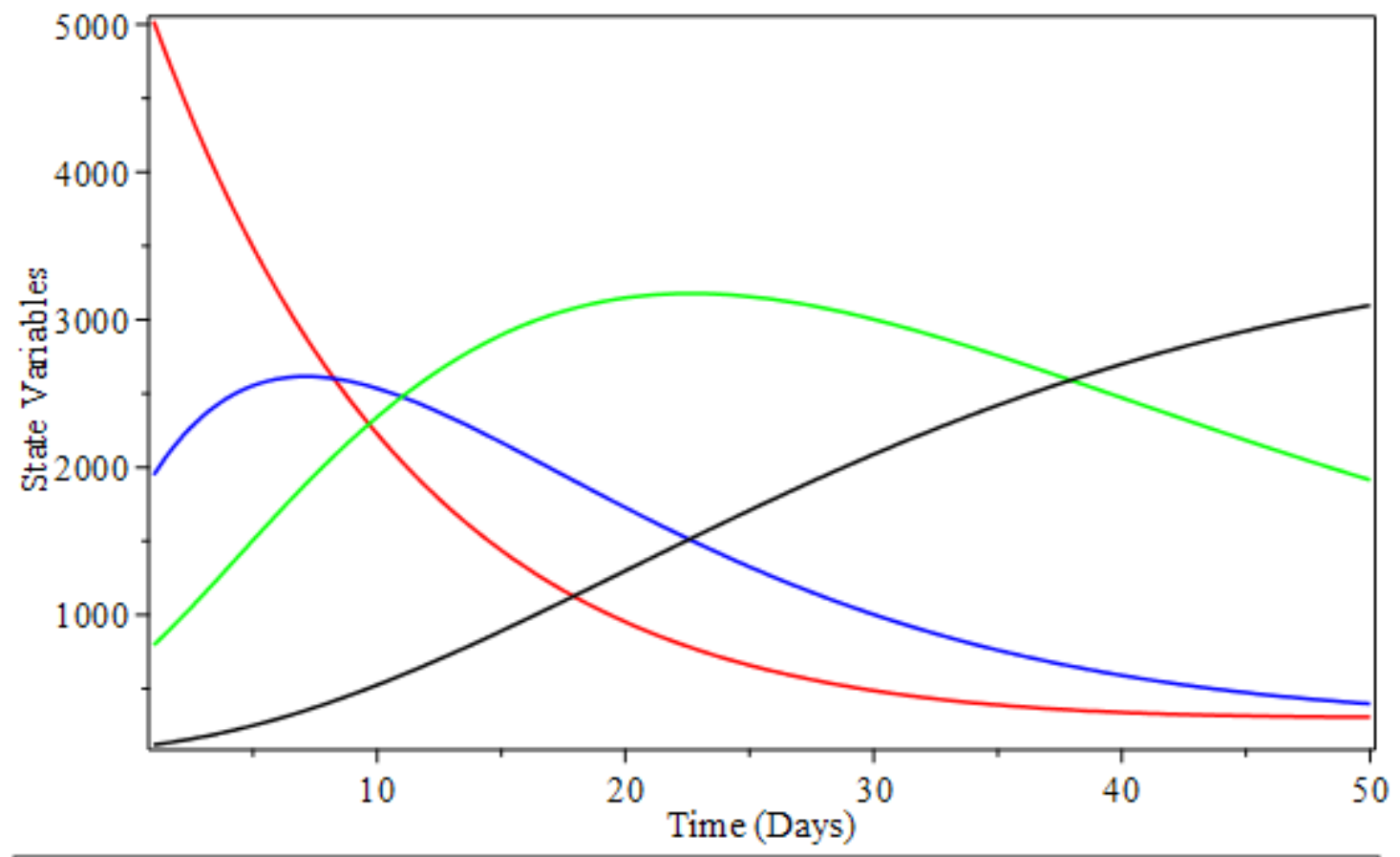

Susceptible Human Sh(t) — Exposed Human Eh(t) - Infected Human Ih(t) Recovered Human Rh(t)

\section{Figure 1}

The dynamical behavior of the human state variables with standard parameter values on the Table 


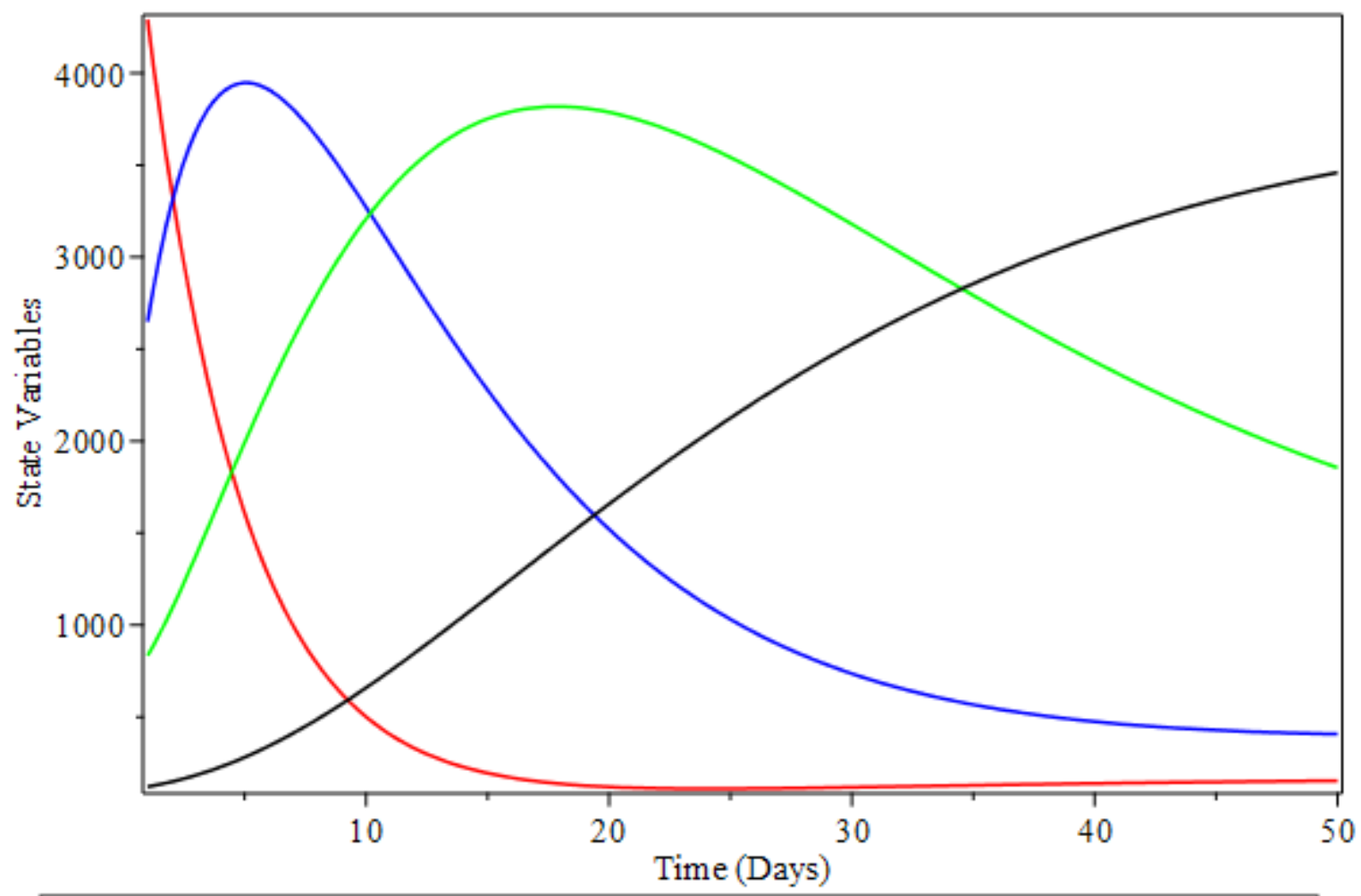

Susceptible Human Sh(t) - Exposed Human Eh(t) - Infected Human Ih(t) Recovered Human Rh(t)

Figure 2

The dynamical behavior of the human state variables when the force of infection and recruitment are varied 


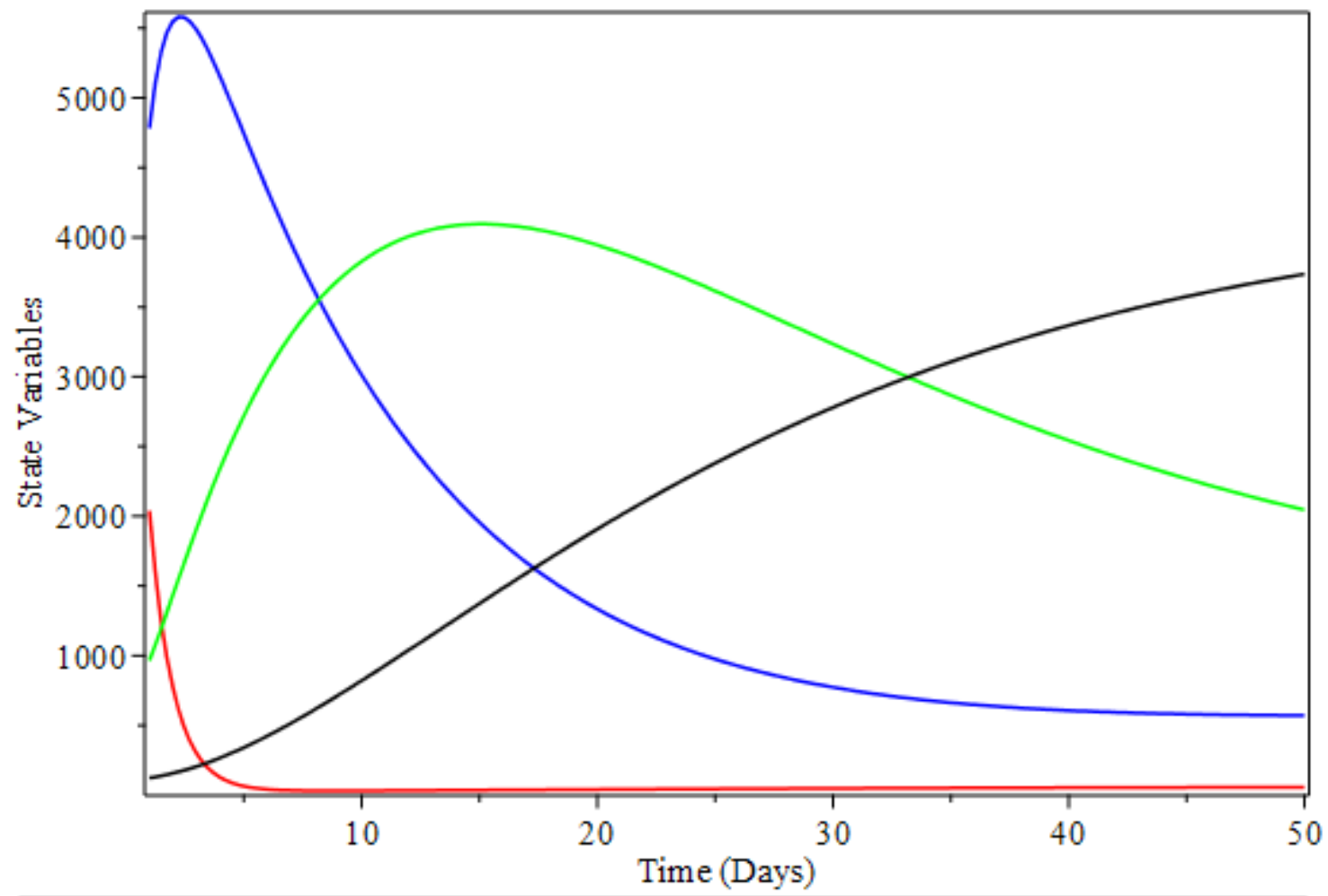

Susceptible Human Sh(t) — Exposed Human Eh(t) — Infected Human Th(t) Recovered Human $\mathrm{Rh}(\mathrm{t})$

\section{Figure 3}

The dynamical behavior of the human state variables when the force of infection is triple and recruitment rate also increases

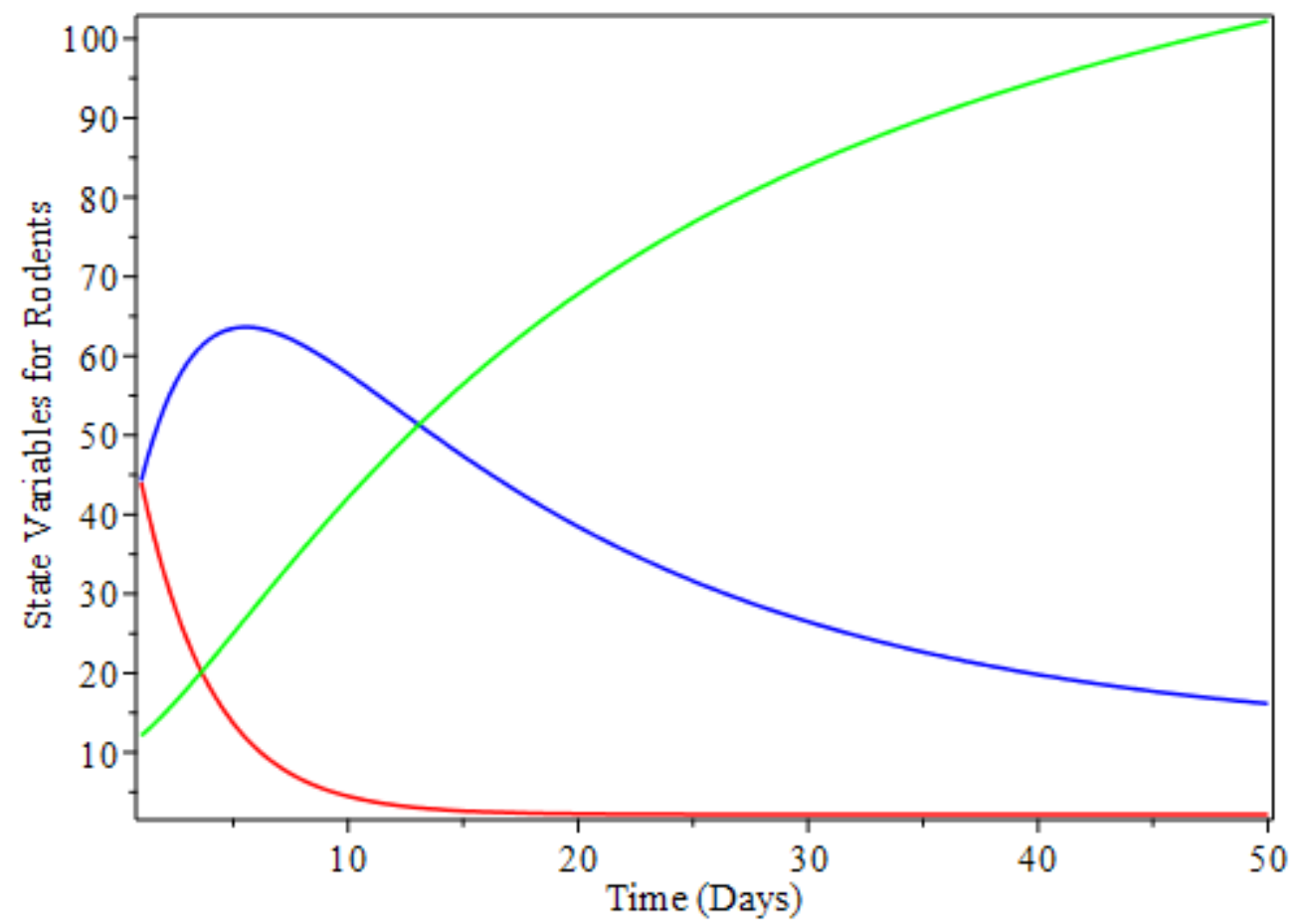

Susceptible Rats $\operatorname{Sr}(\mathrm{t}) \longrightarrow$ Exposed Rats $\operatorname{Er}(\mathrm{t})-$ Infected Rats $\operatorname{Ir}(\mathrm{t})$ 
Figure 4

The dynamical behavior of the Rodents' state variables with standard table values

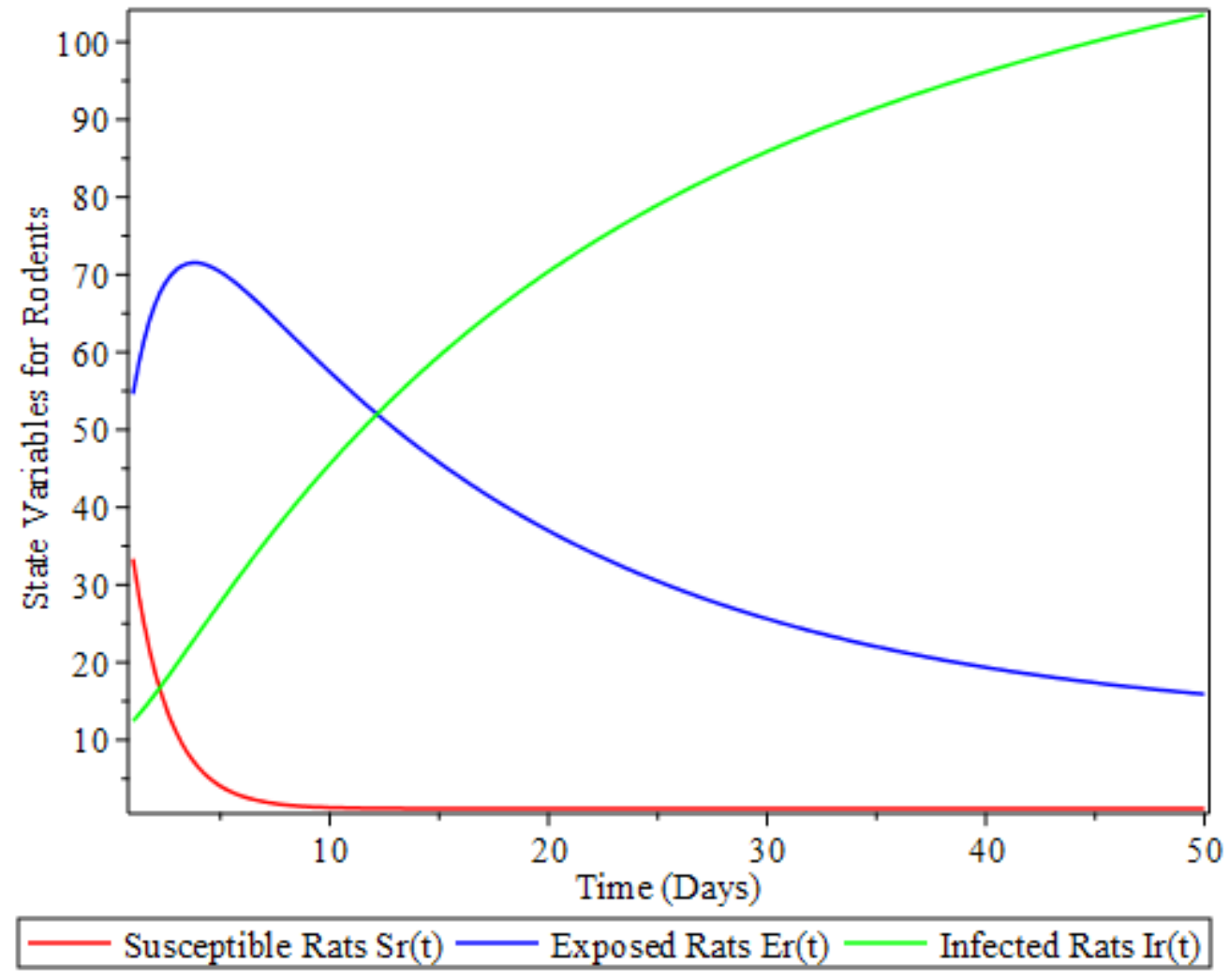

\section{Figure 5}

The dynamical behavior of the Rodents' state variables when the force of infection is tripled 


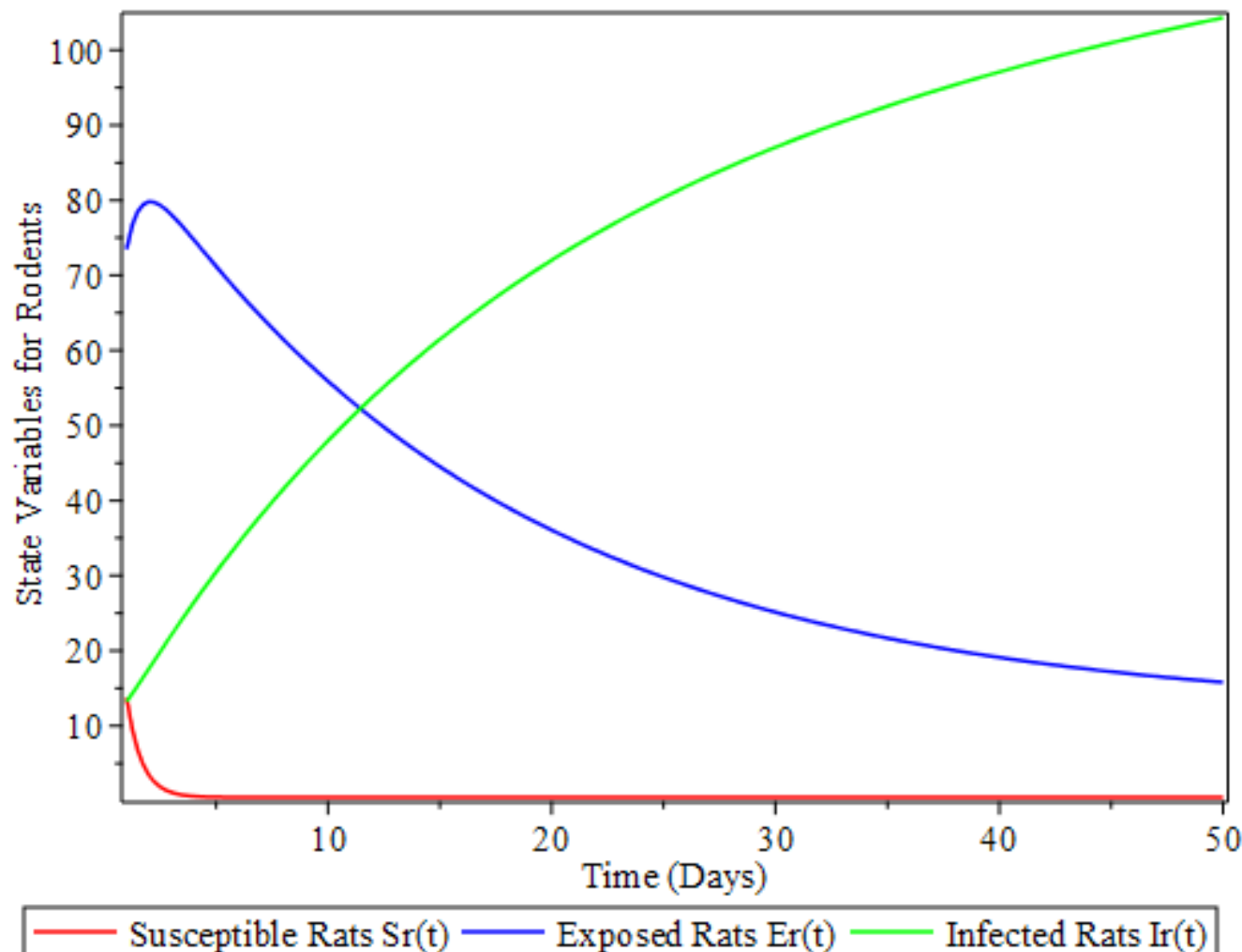

Figure 6

The dynamical behavior of the Rodents' state variables when the force of infection is tripled

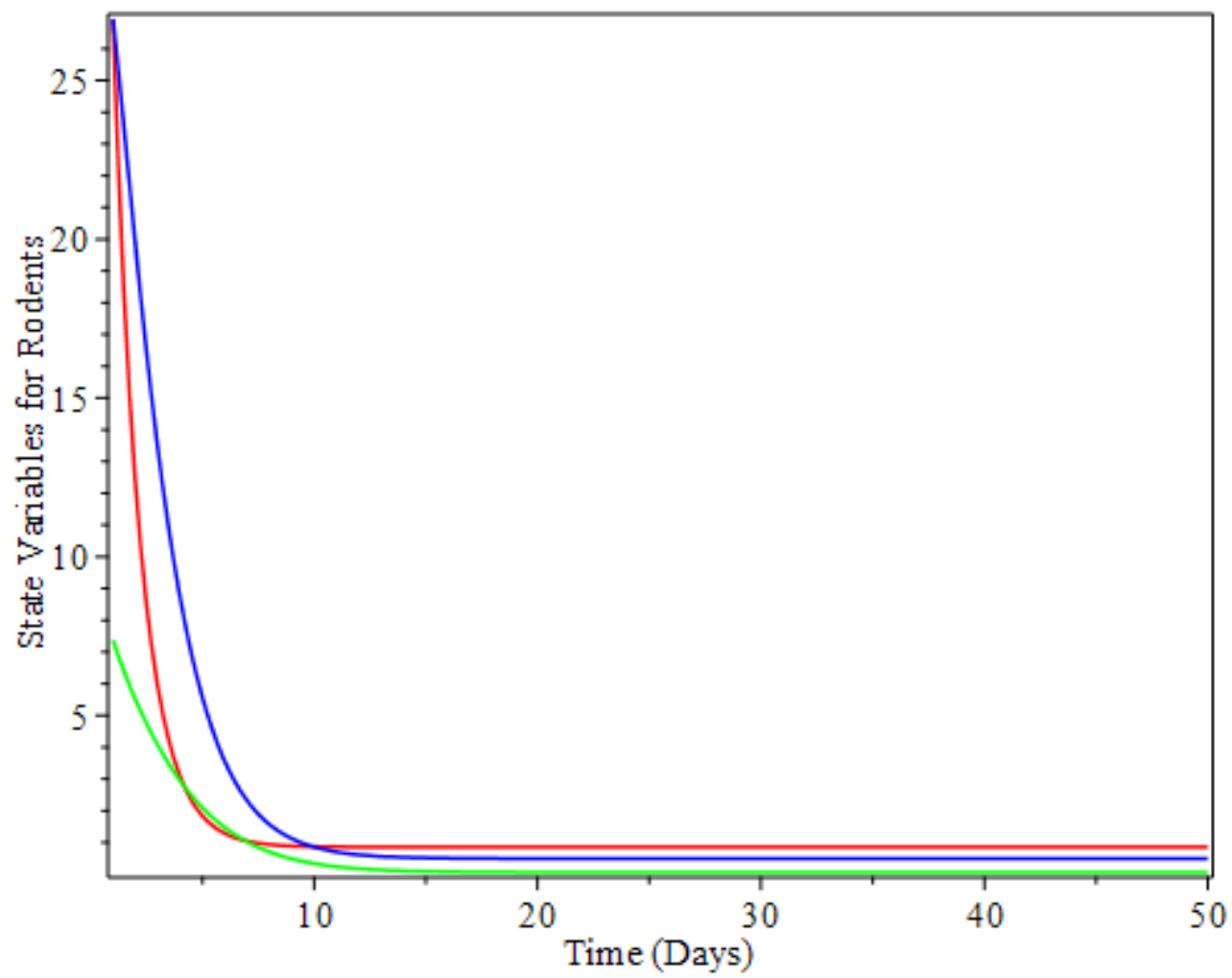

Susceptible Rats $\operatorname{Sr}(\mathrm{t}) \longrightarrow$ Exposed Rats $\operatorname{Er}(\mathrm{t})-$ Infected Rats $\operatorname{Ir}(\mathrm{t})$

Figure 7 
The dynamical behavior of the Rodents' state variables when death rate of rodent is increased

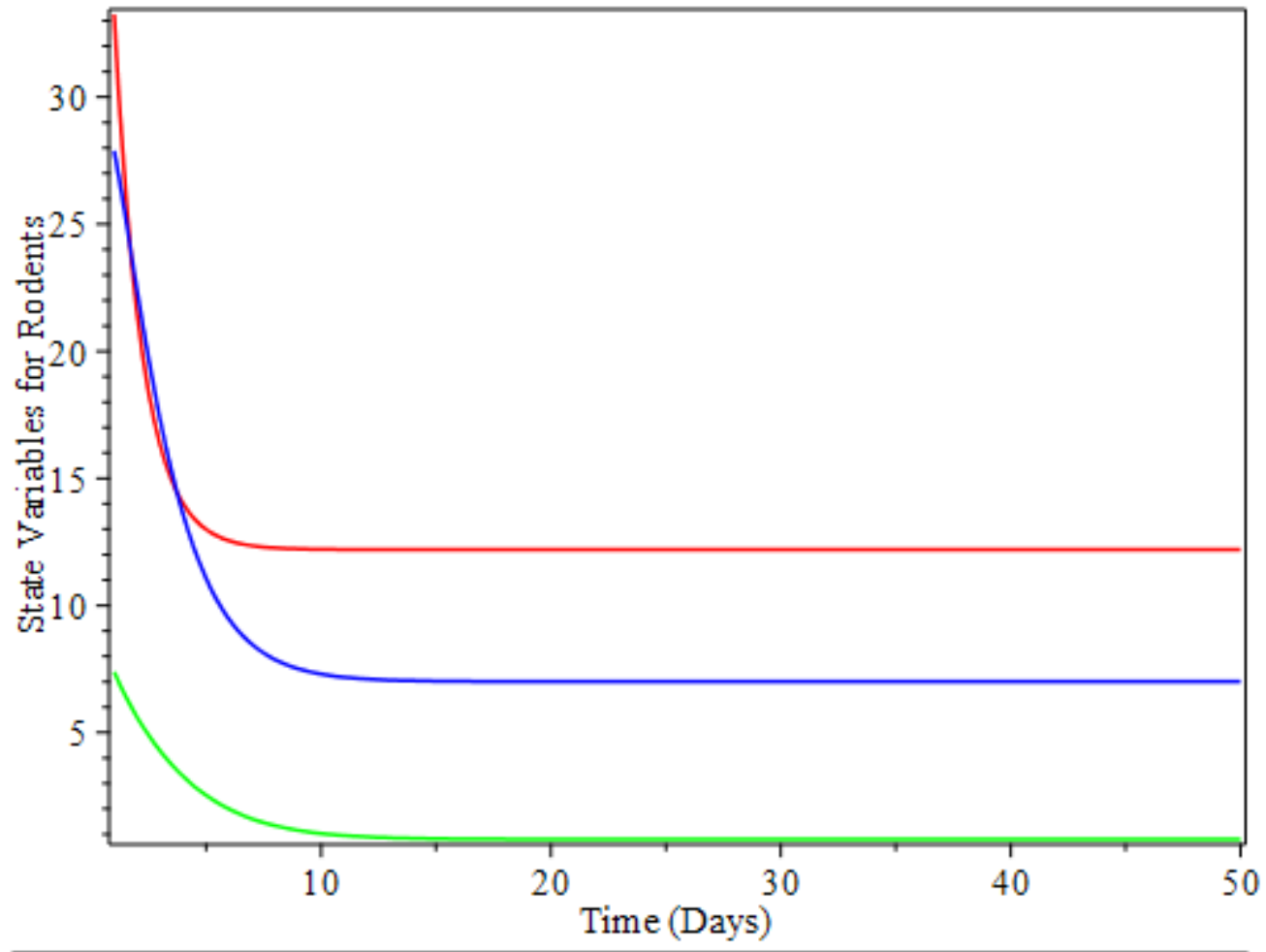

Susceptible Rats $\operatorname{Sr}(\mathrm{t})$ — Exposed Rats Er(t) — Infected Rats $\operatorname{Ir}(\mathrm{t})$

Figure 8

The dynamical behavior of the rodents' state variables when recruitment and death rate is increased

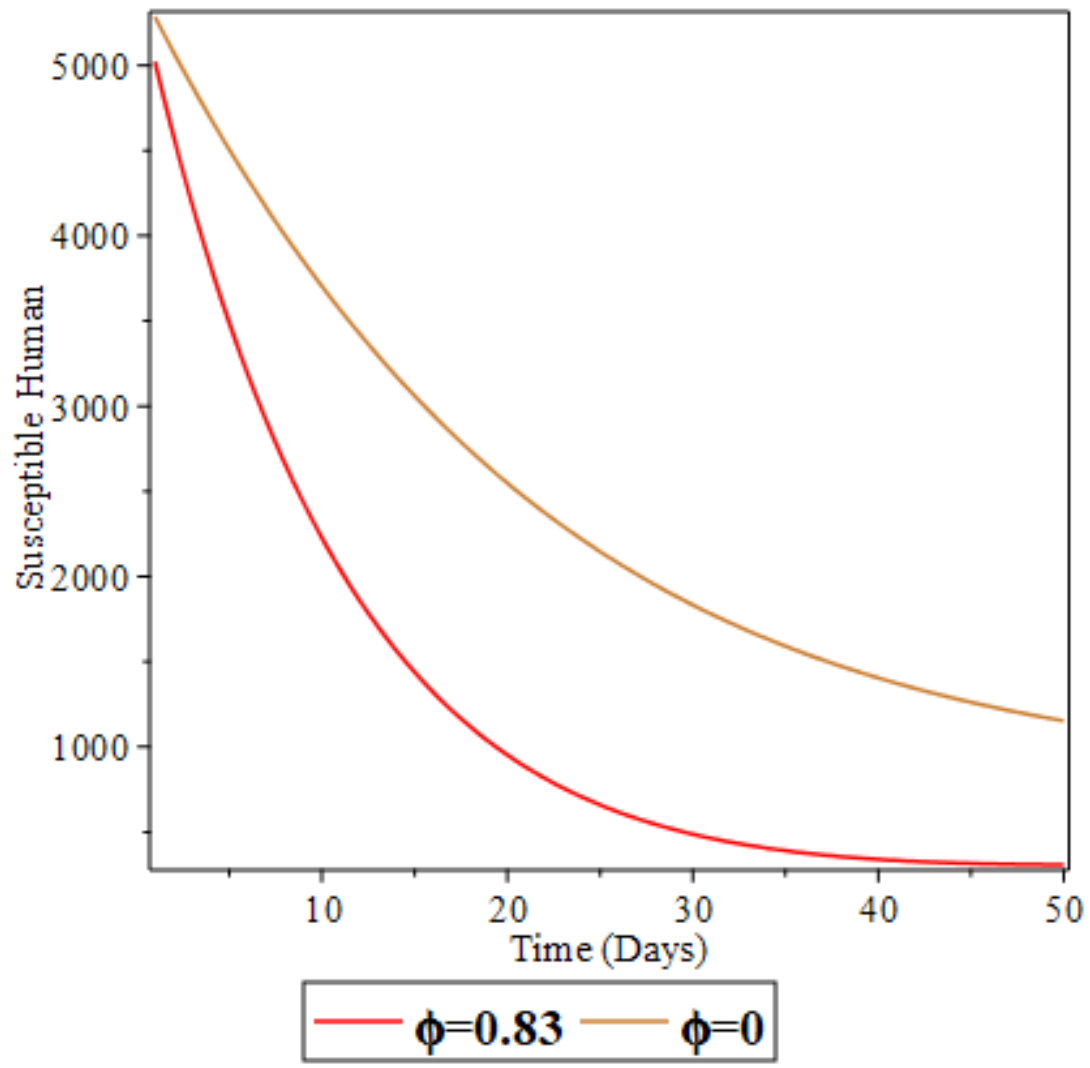


Figure 9

The Changing effect of the susceptible human with and without the "saturation factor".

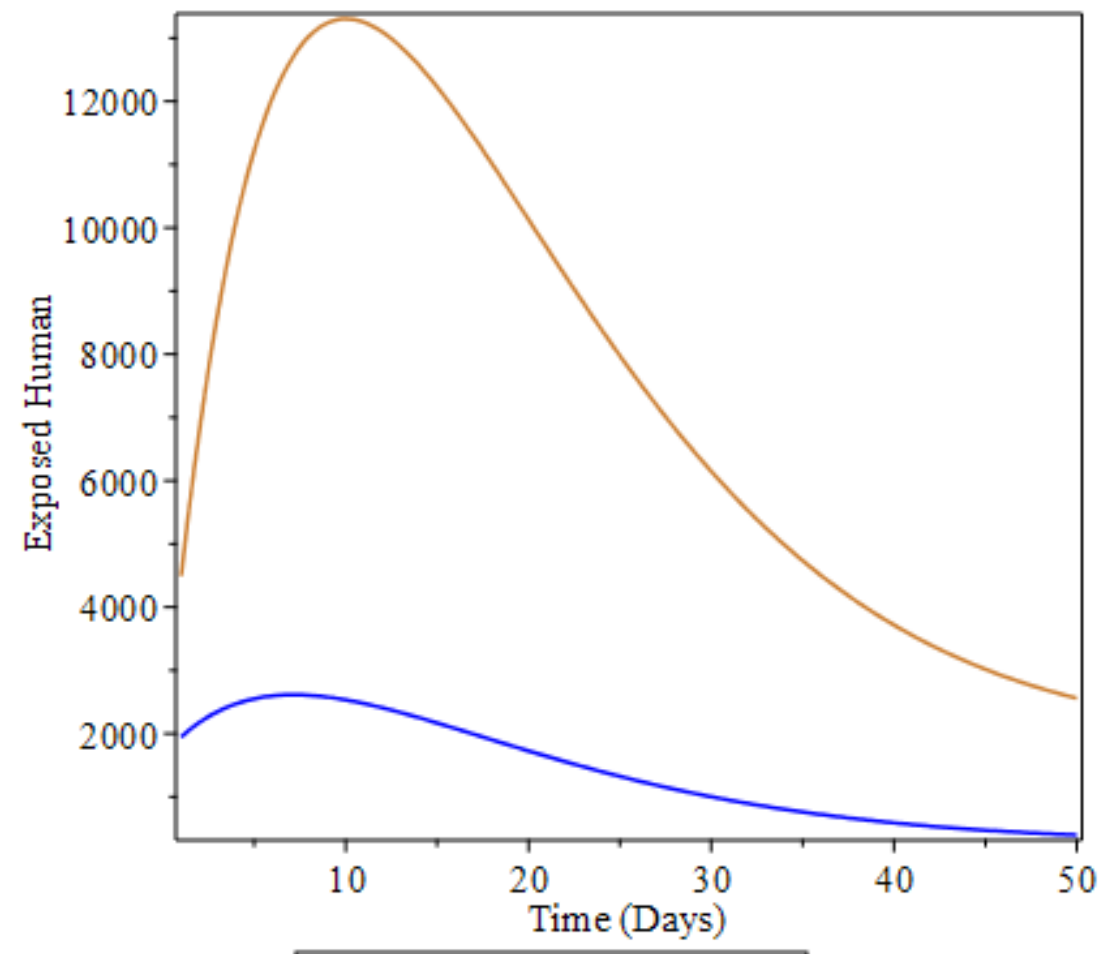

$\phi=0.83-\phi=0.0$

Figure 10

The changing effect of the exposed human with and without the "saturation factor".

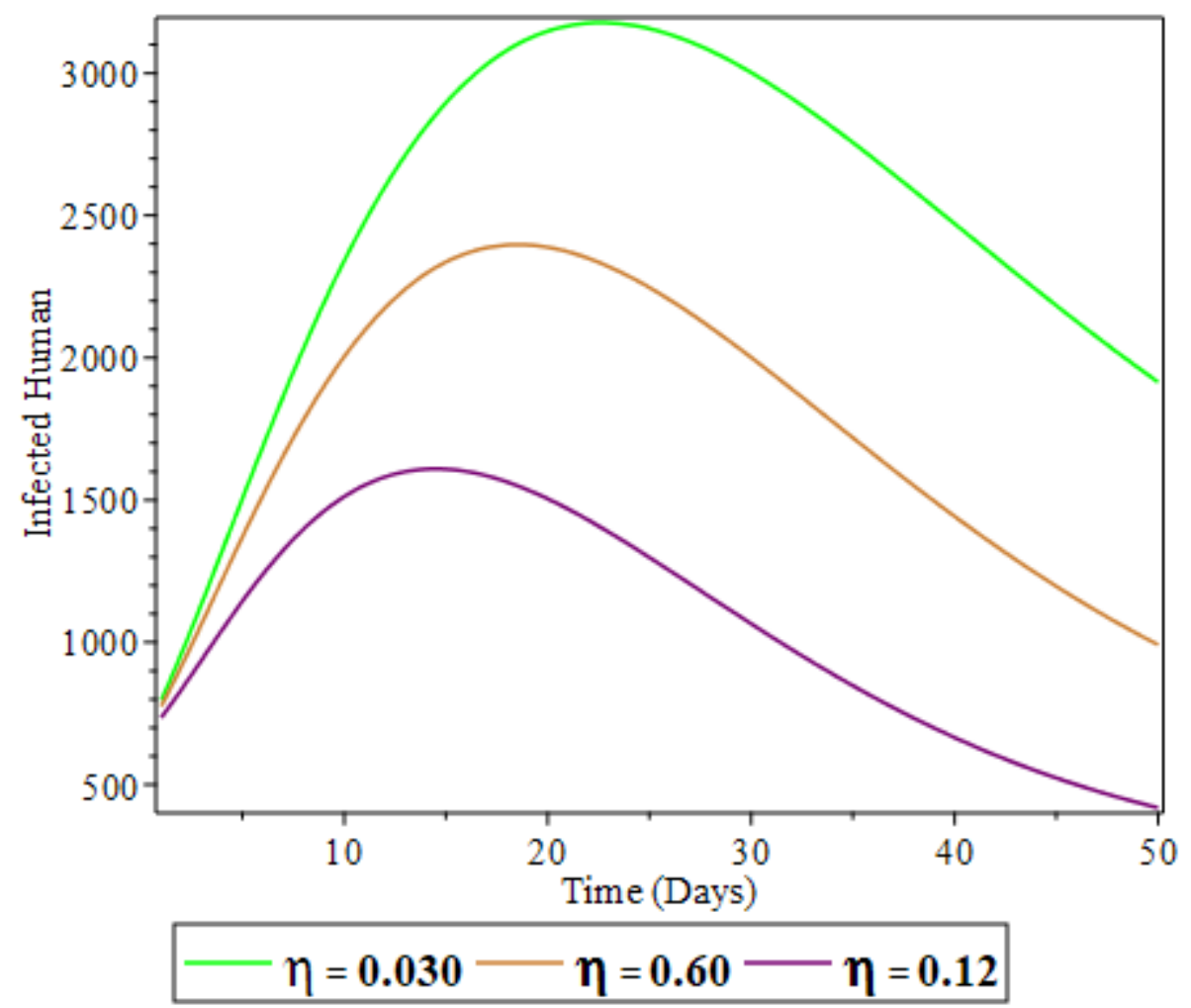


Figure 11

The changing effect of the infected human with increase rate of treatment via the administration of "Ribavirin".

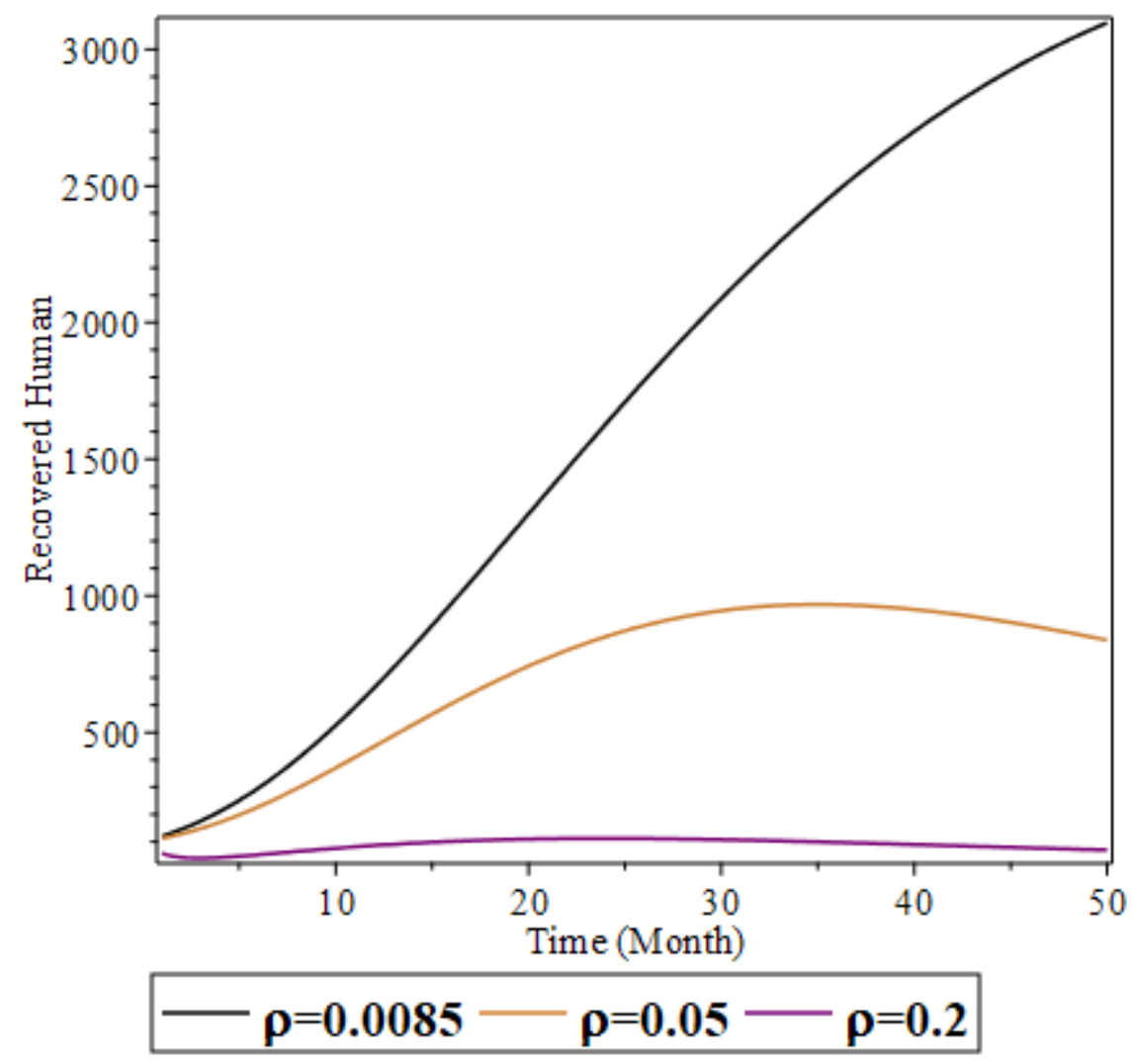

Figure 12

The changing effects on the Recovered human population by varying loss of immunity 


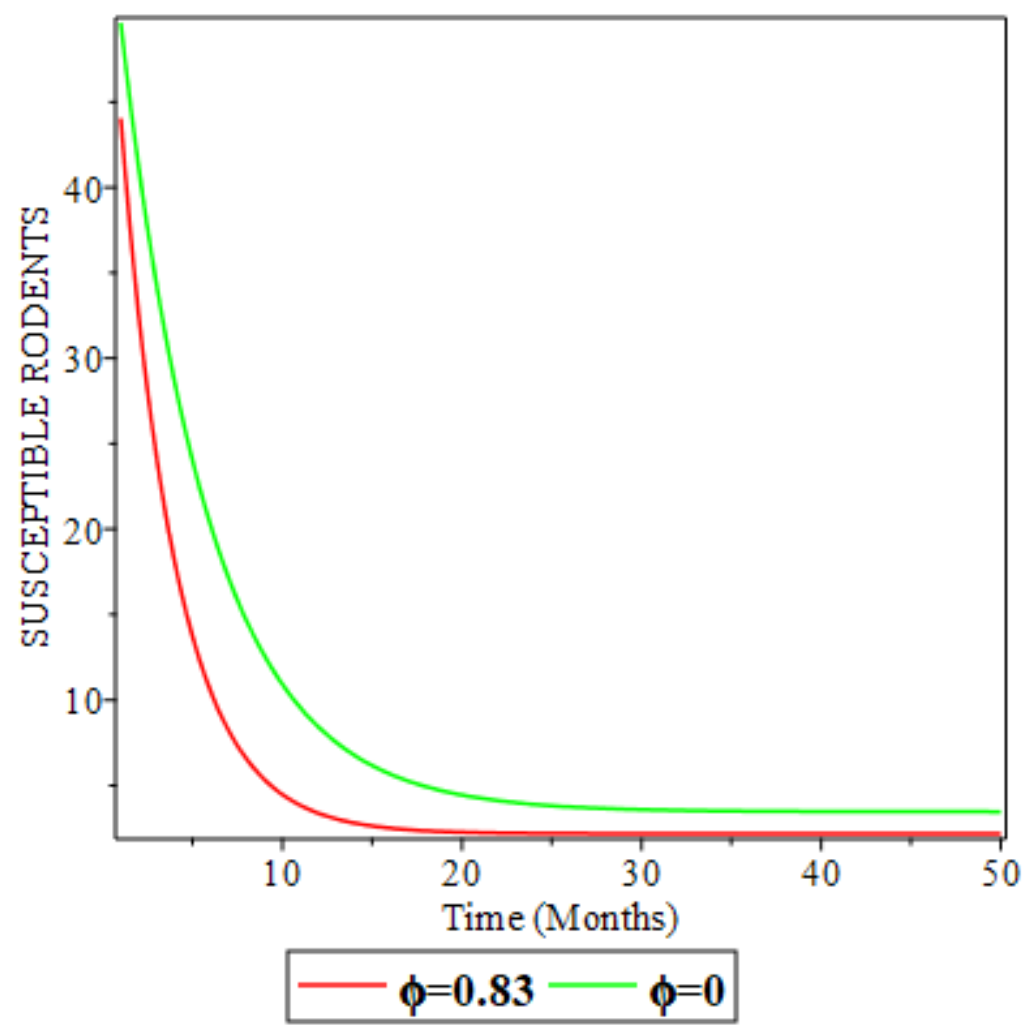

Figure 13

The Changing effect of the susceptible rodents with and without the "saturation factor"

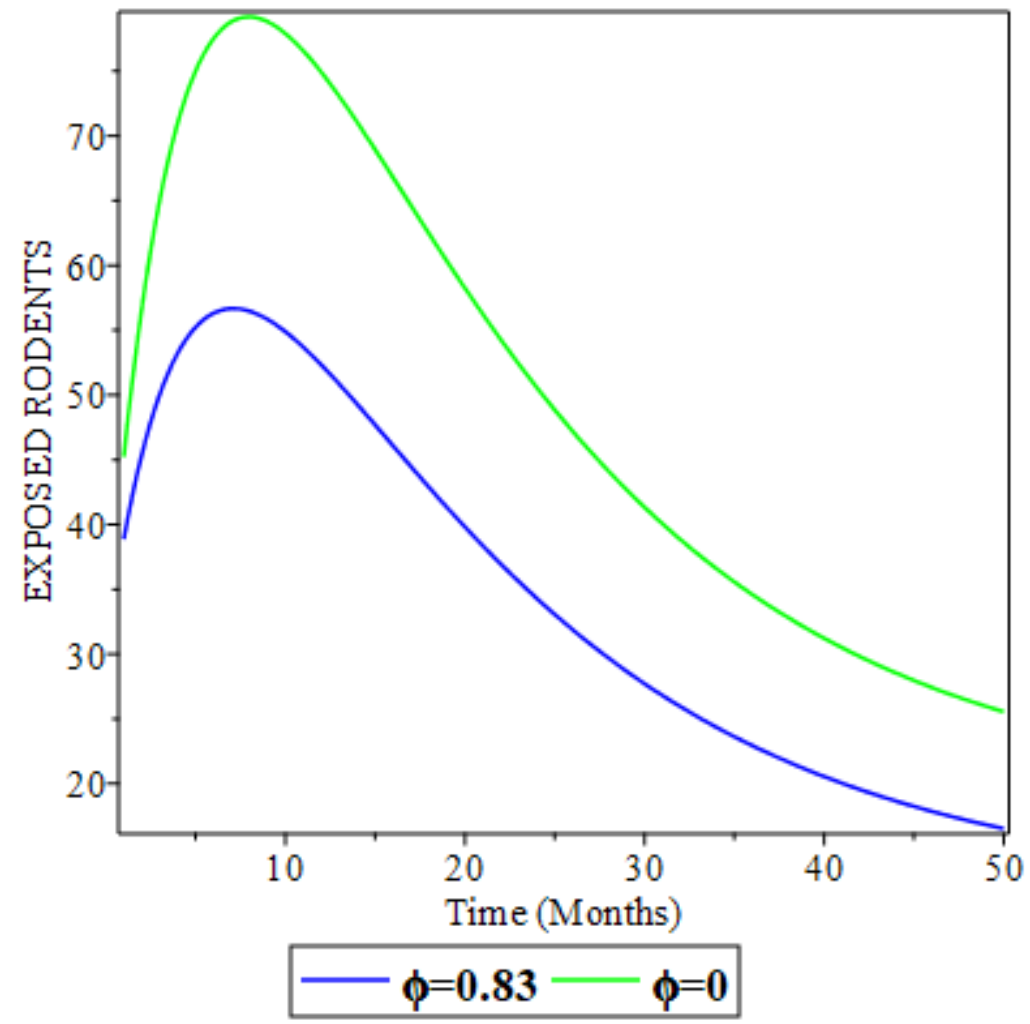

Figure 14 
The changing effect of the exposed rodents with and without "saturation factor"

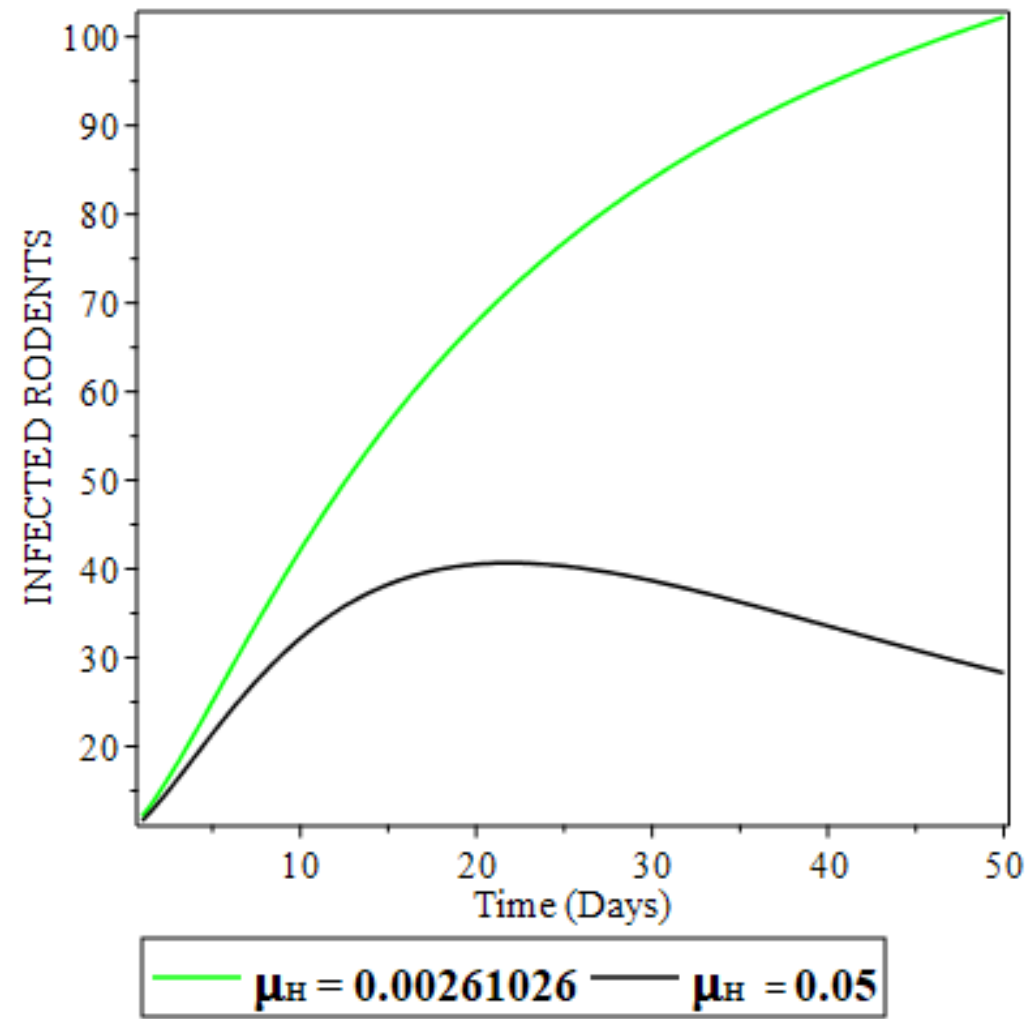

Figure 15

The changing effect of the infected when death rate (by hunting or other control measures, and natural cause) is increased. 